\title{
Title: Cold protection allows local cryotherapy in a clinical-relevant model of
}

\section{traumatic optic neuropathy}

4 Authors: Yikui Zhang ${ }^{1 *}$, Mengyun $\mathrm{Li}^{1 \dagger}$, Bo $\mathrm{Yu}^{1 \dagger}$, Shengjian $\mathrm{Lu}^{1 \dagger}$, Lujie Zhang ${ }^{3 \dagger}$, Senmiao

$5 \mathrm{Zhu}^{1 \dagger}$, Zhonghao $\mathrm{Yu}^{1}$, Tian Xia ${ }^{1}$, Haoliang Huang ${ }^{2}$, WenHao Jiang ${ }^{1}$, Si Zhang ${ }^{1}$, Lanfang Sun ${ }^{1}$,

6 Qian Ye ${ }^{1}$, Jiaying Sun ${ }^{1}$, Hui Zhu ${ }^{1}$, Pingping Huang ${ }^{1}$, Huifeng Hong ${ }^{1}$, Shuaishuai $\mathrm{Yu}^{5}$, Wenjie

$7 \mathrm{Li}^{3}$, Danni $\mathrm{Ai}^{3}$, Jingfan $\mathrm{Fan}^{3}$, Wentao $\mathrm{Li}^{4}$, Hong Song ${ }^{4}$, $\mathrm{Lei} \mathrm{Xu}^{6}$, Xiwen $\mathrm{Chen}^{7}$, Jingxing $\mathrm{Ou}^{8,9}$,

8 Wei $\mathrm{Li}^{10 *}$, Jian Yang ${ }^{3 *}$, Yang $\mathrm{Hu}^{2 *}$, Wencan $\mathrm{Wu}^{1 *}$

9 Affiliations:

$10{ }^{1}$ The Eye Hospital, School of Ophthalmology \& Optometry, Wenzhou Medical University;

11 Wenzhou 325027, China.

$12{ }^{2}$ Department of Ophthalmology, Stanford University School of Medicine; Palo Alto, United

13 States.

$14 \quad{ }^{3}$ Beijing Engineering Research Center of Mixed Reality and Advanced Display, School of

15 Optics and Photonics, Beijing Institute of Technology; Beijing 100081, China.

$16{ }^{4}$ School of Computer Science \& Technology, Beijing Institute of Technology; Beijing 100081,

17 China.

$18 \quad{ }^{5}$ School of Laboratory Medicine and Life Sciences, Wenzhou Medical University; Wenzhou

19325027 , China.

$20 \quad{ }^{6}$ Medical Radiology Department, 2nd Affiliated Hospital, Wenzhou Medical University;

21 Wenzhou 325027, China. 
${ }^{7}$ Animal Facility Center, Wenzhou Medical University; Wenzhou 325027, China.

$23{ }^{8}$ Department of Hepatic Surgery and Liver Transplantation Center of the Third Affiliated

24 Hospital, Guangdong Province Engineering Laboratory for Transplantation Medicine;

25 Guangzhou 510630, China.

$26{ }^{9}$ Guangdong Key Laboratory of Liver Disease Research, the Third Affiliated Hospital of Sun

27 Yat-sen University, Guangzhou 510630, China

${ }^{10}$ Retinal Neurophysiology Section, National Eye Institute, National Institute of Health, NIH;

30 * For correspondence: Yikui Zhang: 86-13705770161, zhang.yikui@wmu.edu.cn, Wei Li: 301-

31 496-6669, liwei2@nei.nih.gov, Yang Hu: huyang@stanford.edu, Jian Yang, jyang@bit.edu.cn,

32 Wencan Wu: wuwencan@wmu.edu.cn.

$33 \uparrow$ These authors contributed equally to this work.

34 Conflict-of-interest statement: The authors have declared that no conflict of interest exists. 


\section{Abstract:}

Therapeutic hypothermia (TH) is potentially an important therapy for central nervous system (CNS) trauma. However, its clinical application remains controversial, hampered by two major factors: 1) Many of the CNS injury sites, such as the optic nerve (ON), are deeply buried, preventing access for local TH. The alternative is to apply TH systemically, which significantly limits the applicable temperature range. 2) Even with possible access for "local refrigeration", cold-induced cellular damage offsets the benefit of $\mathrm{TH}$. Here we present a clinically translatable model of traumatic optic neuropathy (TON) by applying clinical trans-nasal endoscopic surgery to goats and nonhuman primates. This model faithfully recapitulates clinical features of TON such as the injury site (pre-chiasmatic $\mathrm{ON}$ ), the spatiotemporal pattern of neural degeneration, and the accessibility of local treatments with large operating space. We also developed a computer program to simplify the endoscopic procedure and expand this model to other large animal species. Moreover, applying a cold-protective treatment, inspired by our previous hibernation research, enables us to deliver deep hypothermia $\left(4^{\circ} \mathrm{C}\right)$ locally to mitigate inflammation and metabolic stress (indicated by the transcriptomic changes after injury) without cold-induced cellular damage, and confers prominent neuroprotection both structurally and functionally. Intriguingly, neither treatment alone was effective, demonstrating that in situ deep hypothermia combined with hibernation-mimicking cold protection constitutes a breakthrough for TH as a therapy for TON and other CNS traumas. 
Main Text:

56

\section{INTRODUCTION}

Therapeutic hypothermic (TH) has a long history and was firstly described in the Edwin Smith papyrus over 5000 years ago ${ }^{1}$. Although $\mathrm{TH}$ has shown neuroprotection in animal models of central nervous system (CNS) trauma by reducing neuroinflammation and alleviating metabolic demand $^{2}$, its clinical application following CNS injury remains controversial. Randomized controlled clinical trials of TH for traumatic brain injury (TBI) failed to show beneficial effect ${ }^{3-5}$. In spinal cord injury (SCI), TH has not been proven to be neuroprotective in randomized controlled clinical trials, and its effect in animal models is inconsistent ${ }^{2}$.

At least two major factors hamper the clinical application of TH in CNS trauma. First, many CNS injury sites are located deeply, preventing access for local TH. Instead, TH is applied systemically. But systemic drops in body temperature increase the risk of cardiopulmonary disorders, coagulopathy, and electrolyte disturbances ${ }^{6}$, so systemic $\mathrm{TH}$ is limited to 34 and $36^{\circ} \mathrm{C}$. The limited temperature range of TH may account for its inadequate efficacy. Secondly, even if CNS trauma is accessible for local deep hypothermia, the cold destroys neuronal microtubules andmay counteract the benefit of $\mathrm{TH}^{7}$. Our previous study discovered a cold-protective mechanism in hibernators, and found that cold-protective reagents such as protease inhibitors (PI) rescue cold-induced cell damage. If these reagents were applied during local $\mathrm{TH}$, then TH would be a more precise, safe, and effective tool to treat CNS traumas ${ }^{7}$.

The optic nerve (ON), which collects axons from retinal ganglion cells (RGCs), is an ideal CNS tissue to study CNS trauma because it is structurally simple.However, its deep location in the orbit and skull base hampers preclinical tests of local treatment for optic neuropathy (Figure 1A). Minimally invasive trans-nasal endoscopy is widely used in modern neurosurgeries to 
access the distal ON (pre-chiasmatic and chiasmatic $\mathrm{ON}$ ) in case of tumor or optic canal fracture (Figure 1B $)^{8-11}$. The large sphenoid sinus along the distal ON provides sufficient space for local treatment (Figure 1A-C). Here we back-translated clinical trans-nasal endoscopy in large animals to safely expose the distal $\mathrm{ON}$, allowing local damage, observation, and modulation. To facilitate clinical translation, we established a de novo traumatic optic neuropathy (TON) model in goats and non-human primates by performing pre-chiasmatic ON crush. These models recapitulated clinical features of TON such as the injury site, the time course of progression, and the capability of local treatment via trans-nasal endoscopy.

Early transcriptomic changes in the $\mathrm{ON}$ and retina were confined to the injury site and enriched in the pathways of inflammation, ischemia, and metabolism, suggesting that local TH could alleviate secondary damage. To serve the twin goals of administrating local deep cooling therapy and preventing cold-induced microtubule instability, we applied both $\mathrm{TH}$ and cold-protective reagents. Neither treatment had an effect alone. But when combined, local TH and coldprotective reagents achieved significant structural and functional neuroprotection.

Next, we developed a computer program to detail feasible surgical pathways and to optimize the size of endoscopic tools according to a CT scan of the skull. This program was successful in goats, minipigs, beagles, and rhesus macaques. We trust that this software will help other research groups to replicate our novel TH strategy, and will facilitate preclinical tests for other local TON treatments.

This novel combination of TH and a cold-protection strategy can be readily applied not only in TON patients using the same procedures and devices, but also can potentially revolutionize conventional TH for other CNS trauma such as SCI and TBI. Furthermore, this translatable TON model allows pre-clinical testing of local treatments for axonopathy. This may spark a paradigm 
101 shift in the therapeutic approach for TON, so clinicians can move from less effective strategies

102 (e.g. observation alone, or systemic medication; ${ }^{12}$ ) towards more targeted and potentially more

103 effective treatment via minimally invasive trans-nasal endoscopy.

\section{RESULTS}

105

106

107

108

109

\section{Goat is an advantageous species for trans-nasal endoscopic access of the chiasmatic and}

\section{pre-chiasmatic $\mathrm{ON}$}

We first looked for animal species suitable for trans-nasal exposure of the chiasmatic and prechiasmatic ON. Skull computerized tomography (CT) scan showed that the frontal cortex of the domestic pig and beagle (Figure 1D, asterisk) falls between the nasal cavity (Figure 1D, number sign) and the pre-chiasmatic ON (Figure 1D, blue rectangle), thus blocking surgical access. The narrowest portion of rhesus macaque's sphenoid bone body (Figure 1D, red circle) is too narrow (around $2.5 \mathrm{~mm}$ in width) to allow a conventional endoscopic microdrill (2.9mm in diameter) to pass through. On the other hand, the size of the goat's sphenoid bone body (13mm in width) is similar to that of the human's sphenoid sinus (20mm in width), and there is no cortex in between the nasal cavity and the pre-chiasmatic ON (Figure 1D). Additionally, Saanen goats are readily available, easy to raise and handle, genetically editable ${ }^{13}$, and have a genome that is well studied 14-16. Therefore, we decided to use Saanen goat as our research animal model.

\section{Trans-nasal endoscopic exposure of the pre-chiasmatic $\mathrm{ON}$ in goats is feasible and safe}

By using clinically available trans-nasal endoscopes (Supplementary Movie 1), we exposed the goat's sphenoid bone body (Figure 1E), and then removed its anterior cortical bone and inner bone marrow to create an artificial sphenoid sinus. The anterior bony wall of the chiasmatic and 
122 pre-chiasmatic ON laid posteriorly in the sinus (Figure 1F). We then exposed the pre-chiasmatic

123 ON by drilling through the anterior bony wall (Figure 1G, Supplementary Movie 2).

124 To evaluate the safety of trans-nasal endoscopic exposure of the pre-chiasmatic ON, we followed

125 the animal for up to 3 months after surgery. The thickness of the ganglion cell complex (GCC,

126 including RGCs' axons, somas and dendrites) in six areas around the optic nerve head was

127 measured by optical coherence tomography (OCT) at different time points (Figure 1H). There

128 was no significant change in GCC thickness over 3 months after pre-chiasmatic ON exposure in

129 either the surgical eyes (Figure 1I) or the contralateral naïve eyes (Supplementary Figure 1A).

130 In line with the OCT results, both the RGC somal densities and axonal densities were unchanged

131 in the surgical eyes compared to the contralateral naïve eyes at 3 months post surgery ( $3 \mathrm{mps}$ )

132 (Figure 1J, K). Consistent with morphological studies, functional readouts (pupillary light reflex

133 (PLR), pattern electroretinogram (PERG), flash visual evoked potential (FVEP) tests) of the

134 surgical eyes were not changed over 3 mps compared with the baseline (Supplementary

135 Figures 1B, C, D). Taken together, these results show that endoscopic exposure of the pre-

136 chiasmatic $\mathrm{ON}$ is feasible and safe.

\section{Trans-nasal endoscopy-mediated pre-chiasmatic ON crush causes visual loss in goat}

138 Most human TON occur in the pre-chiasmatic ON due to force transmission (Figure 1B $)^{11,17}$. To

139 establish a clinically relevant animal model of TON which enables local modulation of the

140 injured pre-chiasmatic ON, we performed pre-chiasmatic ON crush in goat using a trans-nasal

141 endoscopic approach. As illustrated in Figure 2A, we first drilled the anterior bony wall to

142 expose the pre-chiasmatic $\mathrm{ON}$, and then manually compressed the pre-chiasmatic ON with the

143 blunt blade of a periosteal elevator until it met the optic canal's posterior bony wall (Figure 2B,

144 Supplementary Movie 3). 
The visual function of the injured eye was lost after pre-chiasmatic ON crush. Both the direct PLR (dPLR) in the injured eyes and the indirect PLR (iPLR) in the contralateral control eyes disappeared after injury (Figure 2C), whereas the dPLR in the contralateral eyes and the iPLR in the injured eyes remained intact (Supplementary Figure 2A, left panel). These results suggested that $\mathrm{ON}$ function in the injured eyes was lost whereas the contralateral ON was still functional; additionally, the oculo-motor nerves of both eyes were unaffected by pre-chiasmatic ON crush ${ }^{18}$. Consistently, the ratio of the FVEP P1-N1 and P2-N1 amplitudes of the injured eyes to the contralateral eyes decreased significantly compared to the baselines (Figure 2D), indicating impairment of the retino-geniculate pathway ${ }^{19}$. Interestingly, the ratio of PERG P1-N1 amplitude of the injured eyes to that of the contralateral eyes remained unchanged at 1 week post injury ( 1 wpi), then decreased significantly at 1,2 and 3 months post injury (mpi) compared to the baseline (Figure 2E), indicating that RGC function degraded over time ${ }^{20}$.

Taken together, the visual deficits (PLR, PERG and FVEP abnormality) presented in the TON model are similar to the clinical manifestations of patients with monocular TON (i.e., Marcus Gunn pupil, loss of visual acuity, and reduction in VEP signals) ${ }^{21}$.

\section{Progressive RGC and ON degeneration in goat TON model}

The GCC thickness ratio of the injured eyes to contralateral control eyes remained stable at 1 wpi, then decreased progressively over 3 mpi (about $89 \%, 81 \%, 74 \%$ of the contralateral eyes at 1, 2, 3 mpi, respectively) (Figure 3A). The GCC thickness in the contralateral eyes was unchanged after ON crush (Supplementary Figure 2A, right panel). Progressive GCC thinning occurred in all six areas around the $\mathrm{ON}$ head (Figure 3A), indicating that ON crush caused widespread axonal damage with no quadrant being spared. Importantly, the time course of GCC thinning in our model closely resembles that of TON patients ${ }^{22,23}$. 
There was no significant RGC soma loss at $1 \mathrm{mpi}$, yet the RBPMS (RNA-binding protein with multiple splicing) immunoreactivity in some RGCs was weak and irregular (Figure 3B). At 3 mpi, only $25 \%$ of RGCs survived in the injured eyes compared with the contralateral eyes (Figure 3B). RGC density in the contralateral eyes remained stable at 1 and $3 \mathrm{mpi}$ (Supplementary Figure 2B, left panel). It is worth noticing that our previous study in goat showed that retrobulbar ON crush resulted in more severe RGC loss at 3 mpi (less than 5\% survival $)^{24}$.

To study the temporal and spatial patterns of axonal degeneration after pre-chiasmatic ON crush, we examined semi-thin cross-sections from four different sites of the ONs (named \#1, 2, 3, 4) of both eyes at 1 and $3 \mathrm{mpi}$ (Figure 3C). For the contralateral ON, the surviving axonal densities remained statistically the same at 1 and $3 \mathrm{mpi}$, and there was no significant difference in axonal densities among different ON sites (Supplementary Figure 2B, right panel). For the injured eyes, there were few surviving axons at distal regions (\#3, \#4) at 1 and 3 mpi. In contrast, axonal densities of the proximal ON segments (\#1, \#2) of the injured eyes were almost intact at $1 \mathrm{mpi}$ and then decreased dramatically at $3 \mathrm{mpi}$ (Figure 3C), indicating progressive retrograde axonal degeneration, which was also observed in rodent models of distal ON injury ${ }^{25}$.

Taken together, these morphological studies demonstrate progressive retrograde neural degeneration in our TON model, the time course of which is similar to that of clinical TON.

\section{Transcriptomic analysis revealed early changes in ischemia, inflammation, and metabolic}

\section{pathways at the injured optic nerve}

To explore early changes and identify potential therapeutic targets in the injured eye, we sampled from the retina $(\mathrm{R})$, proximal (\#1) and pre-chiasmatic (\#2) ON segments in both the injured eye and the contralateral eye at 1 day post injury (dpi), and performed RNA-sequencing (Figure 4A). 
Compared with the contralateral eye, there were few differentially expressed genes (DEGs) in

192

193

194

195

196

197

198

199

the retina and proximal $\mathrm{ON}$ of the injured eye, indicating the transcriptomic status and microenvironment of the retinal and proximal axonal were stable at 1 dpi (Figure 4B). In addition, DEGs between the proximal and pre-chiasmatic $\mathrm{ON}$ of the contralateral eye were rare, suggesting that the axonal status and microenvironment were consistent between different ON segments (Figure 4B). In contrast, there were large amount of DEGs in the injured ON segment (Inj_\#3) compared with either the contralateral pre-chiasmatic ON (ctrl_\#3) or the ipsilateral proximal ON (Inj_\#1). To narrow down the ranges of target genes, we extracted overlapping DEGs between Inj_\#3 vs. Ctrl_\#3 and Inj_\#3 vs. Inj_\#1, and found that 91\% of the overlapping DEGs were clustered in the pathways of inflammation, ischemia, and metabolism (Figure 4D, E, Figure S3A). We further found that most of the DEGs with high connectivity ("hub genes") were also enriched in these pathways (Figure S4A, B, D), indicating that targeting these pathways with local treatment could alleviate secondary damage. Gene ontology (GO) analysis of the overlapping DEGs and hub genes is listed in Figure S3B, S4C.

\section{Protease inhibitors (PI) rescued cold-induced axonal microtubule damage}

Hypothermic therapy can alleviate inflammation and reduce metabolic demand after CNS

trauma $^{2}$. However, our previous study showed that the cold destroys neuronal microtubules, and that microtubules can be rescued by cold-protective reagents such as $\mathrm{PI}^{7}$. In this study, goat retinal explants cultured at $0^{\circ} \mathrm{C}$ suffered severe axonal damage compared to those cultured at $37^{\circ} \mathrm{C}$, as shown by beta- 3 tubulin-positive axonal length and axonal beads. When we added PI into $0^{\circ} \mathrm{C}$ medium, this cold-induced axonal degradation was significantly reduced (Figure 5AD). 

chiasmatic ON crush

To prevent axons from degrading during local TH, we applied PI to the injured pre-chiasmatic ON while administering hypothermia locally (Figures 5E, F, Supplementary Movie 4). The temperature curves of the cooling water and the cling film over time are shown in Figure 5G.

218 The sponge placed at the injury site was filled with goat serum with or without PI.

219 We found that local combinatory delivery of hypothermia and PI significantly alleviated neural

220 degeneration compared with the no treatment group at 1 mpi in terms of PERG amplitude, GCC

221 thickness and axonal density at the injury site (Figure 5H-J). In contrast, local hypothermia or

222 local PI alone failed to show significant neural protection in either PERG signal or GCC

223 thickness (Figure 5H, I).

224 Neither FVEP or PLR were rescued in any treatment group (Supplementary Figure 5A-C),

225 since both require an intact ON. In the combinatory treatment group, there were few residual 226 axons at the \#4 ON segment (distal to the injury site) (Supplementary Figure 5D), indicating

227 Wallerian degeneration was not significantly rescued.

228 Computer program-aided optimization of trans-nasal endoscopic surgery in multiple large 229 animal species

230 To simplify the trans-nasal endoscopic surgery and expand the approach to other large animal 231 species, we developed a computer program to generate virtual surgical paths. The flowchart of 232 programming is detailed in Figure 6A. Briefly, we input CT scans of the animal's skull into the 233 program to outline the surgical space, the non-surgical space, and the surgical target (Figure 
6B). Then, we entered the size of the surgical microdrill to generate the surgical corridor (Figure 6C).

As shown in Figure 6D, there were five degrees of freedom for the surgical corridor when its head met the target (optic canal): rotation around $\mathrm{x}, \mathrm{y}, \mathrm{z}$ axes; spin around the body (major axis); and movement along the neck. We set the step size and ranges of rotation (around $\mathrm{x}, \mathrm{y}, \mathrm{z}$ axes) as $5^{\circ}$ and $180^{\circ}$, the step size and ranges of spin as $5^{\circ}$ and $360^{\circ}$, the step size and range of movement as $1 \mathrm{~mm}$ and $15 \mathrm{~mm}$. Therefore, the total number of theoretical paths were calculated as $(180 / 5) *(180 / 5) *(180 / 5) *(360 / 5) *(15 / 1)=50388480$. The computer program tried all these theoretical paths to find collision-free surgical corridors within the surgical space. In another words, no pixel of the surgical corridor should be included in the non-surgical space. For example, in Figure 6E, the surgical corridor on the right collided with the orbit (non-surgical space, shown in red), so this path was excluded. If there was no feasible surgical pathway with a given size of microdrill, the computer program would reduce the size of the microdrill, and repeat the searching process.

Figure 6F-I shows the virtual surgical paths generated by the computer program to access the pre-chiasmatic $\mathrm{ON}$ in goat, beagle, domestic pig and rhesus macaque, respectively. The surgery

251 horizontal plane of the CT scan (middle panel). The right panels demonstrate the final position of the surgical microdrill when its head meets the target (pre-chiasmatic ON in the optic canal).

253 The step-by-step surgical path plans for goat, beagle, domestic pig and rhesus macaque are presented in Supplementary Movies 5-8. 
to the pre-chiasmatic ON (Figure 1D), it can be circumvented either inferiorly or laterally

(Figure 6G, H), and (3) in rhesus macaques, we can access the pre-chiasmatic ON by either circumventing the narrowest portion of the sphenoid bone body (Figure 6I) or using a smaller surgical microdrill (2.5 $\mathrm{mm}$ in diameter) (Figure 6K).

This computer-assisted surgical path planning program can be used to (1) screen suitable animals

262 for trans-nasal endoscopy before surgery, (2) provide a detailed roadmap for endoscopic surgery,

263 and (3) potentially pave the way for robotic surgery to industrialize this novel TON model. optimization

The visual systems of non-human primates (including rhesus macaques) resemble those of humans in terms of macular structure, RGC subtypes, and ON projection pattern ${ }^{26}$. Therefore, we decided to expand our modeling to rhesus macaques. Directly informed by our computer program, we successfully exposed the pre-chiasmatic ON in rhesus macaque via trans-nasal endoscopy (Supplementary Movie 9), confirmed the target with the surgical navigation system (Figure S6A), and performed pre-chiasmatic ON crush (Figure S6B).

We observed substantial visual loss and neural degeneration following crush injury. The ratios of

274 eyes fell significantly compared with baseline (Figure S6C, D). Structurally, GCC thickness in

275 the contralateral eyes did not change over time (Supplementary Figure 2C), whereas

276 progressive thinning of GCC was observed in the injured eyes (Figure S6E). At 3 mpi, the RGC

277 soma density and the axonal densities at different ON sites of the injured eyes decreased

278 dramatically compared with the contralateral eyes (Figures S6F, G). 


\section{DISCUSSION}

In this study, we developed a novel TH strategy on a de novo, translatable, large animal model of CNS axonopathy. Briefly, we back-translated clinical trans-nasal endoscopic surgery into goats and non-human primates to establish a translatable TON model by crushing the pre-chiasmatic ON. This model faithfully resembles the key features of clinical TON, including specific injury site, spatiotemporal pattern of neurodegeneration, and the capability to locally modulate the injured pre-chiasmatic ON. RNA sequencing revealed that early changes were confined to the primary injury site and were enriched in inflammation, ischemia, and metabolism. Local codelivery of hypothermia with cold-protective reagents showed significant structural and functional neuroprotection, but neither treatment was neuroprotective by itself. To ensure other research groups can faithfully replicate this endoscopy (for TH or for other ON treatments), we made a computer program to optimize surgical paths based on skull CT scans. This study potentially revolutionizes TH for CNS traumas, and provides translatable large animal models for developing local therapeutic strategies for TON and other axonal injuries.

\section{Current treatment for TON}

TON causes severe visual loss after head trauma. Currently, no treatment for TON has been proven effective by randomized controlled trials; mostly, mere observation is recommended ${ }^{21,27}$. In situ manipulation of the micro-environment of injured CNS axons has proven beneficial in SCI model and peripheral nerve graft transplantation studies ${ }^{28,29}$. However, most of the ON is hard to expose due to its deep location and crowding by neighboring tissues. Drugs delivered systemically or regionally (i.e., intraocularly, intra-orbitally, etc.) can hardly reach the injured distal ON at sufficient and stable concentrations due to the blood brain barrier, diffusion/dilution, and impaired axonal microvasculature ${ }^{30-32}$. Our TON model allows clinically-resembling local 
treatment via trans-nasal endoscopy with a large operating space. This foundation may trigger a paradigm shift for TON therapies away from mere observation or systemic steroid administration, and towards promising endoscopic treatment.

\section{Hypothermic therapy and cold-protective strategies}

Systemic hypothermia may cause coagulopathy, electrolyte disturbances, myocardial ischemia, atrial fibrillation, sepsis, pneumonia, and altered drug metabolism ${ }^{6}$. Instead, we targetted hypothermia to the injured pre-chiasmatic ON to prevent early changes in ischemia, inflammation, and metabolism transcripts (as revealed by RNA-sequencing at $1 \mathrm{dpi}$ ).

Although hypothermic therapy was tested as a treatment for CNS trauma for many years, it has not shown neuroprotective effects in randomized controlled trials of TBI or $\mathrm{SCI}^{2,33}$. It is unclear why TH was ineffective, but cold-induced neural damage may counteract any therapeutic effects.

Our recent work showed that the cold degrades neuronal microtubules, but neurons can be spared with reagents such as $\mathrm{PI}^{7}$. In this study, neurons were protected when TH was combined with PI. Neither treatment alone was effective. This novel combinatory strategy can be readily applied to TON patients using established endoscopic procedures, reviving TH as a therapy for TON and other CNS traumas.

\section{Advantages of our TON model compared with other TON models}

(1) Clinical translatability: Compared with the conventional retrobulbar ON crush model, our model with pre-chiasmatic ON injury resembles key clinical features of TON such as the specific injury site and the time course of neural degeneration, and allows local trans-nasal treatment using clinical endoscopic procedures and tools. As such, the pre-clinical tests of local treatment for TON succeeded in our model, and can be readily translated to TON patients. Recently, rodent 
models have been developed in which ultrasound or impact triggers distal ON injury, but unwanted collateral damage is usually inevitable in these models ${ }^{34-36}$. Besides, clinically translatable, local treatment of injured ONs via trans-nasal endoscopy cannot be performed in these rodent models.

Non-human primates including rhesus macaques have human-like visual systems and pathophysiological responses to CNS injury which do not exist in other mammals ${ }^{26,37,38}$. Our work provides goat and non-human primate TON models to meet different experimental needs and budget plans for mechanistic studies or pre-clinical tests of effective treatments.

\section{(2) Accessibility to the injury site within a large operating space (sphenoid sinus): In this}

study, histological and transcriptomic analyses found that axonal damage was initially confined to the primary injury site. The affected pathways (ischemia, inflammation, and metabolism) offer potential therapeutic targets. In our model, the chiasmatic and pre-chiasmatic ON can be exposed safely under direct visual guidance within the spacious sphenoid sinus, facilitating in situ damage, observation, and treatment of the ON with complex devices.

Traditional retrobulbar approach in rodent model allows access only to the most proximal ON within the crowded orbit. Furthermore, exposure of the retrobulbar $\mathrm{ON}$ is much harder in human or large animal models, and requires lateral orbitotomy, which causes transient orbital edema and retinal dysfunction ${ }^{39}$. Although access to the distal ON can be achieved by a conventional intracranial approach with corticectomy or a recently developed intra-orbital approach by blind injection, these approaches may cause unwanted collateral damage to the important adjacent tissues $^{40}$. In addition, the operating space is restricted in these approaches due to crowding in the cranial cavity or orbital apex. 
(3) Facilitation eye-brain reconnection: According to our study, the axonal density of the intraorbital ON segment remained statistically intact $1 \mathrm{mpi}$; therefore, $\mathrm{ON}$ regeneration can start from the pre-chiasmatic ON segment. On the contrary, the traditional retrobulbar ON crush model requires a much longer $\mathrm{ON}$ regeneration distance to reconnect with the brain (i.e., the distance is doubled compared with our TON model), making functional regeneration extremely challenging $^{41}$. In addition, our TON model allows local modulation of the microenvironment of the pre-chiasmatic and chiasmatic $\mathrm{ON}$ to fuel and guide $\mathrm{ON}$ regeneration.

\section{Limitations of this study}

One limitation of this study is a small sample size in each therapeutic group. We were aware that an analysis of sample size and power were needed for each comparison. However, due to ethical issue and limitations of housing space and other resources, we only used 3-6 goats/rhesus macaques in each group according to our previous experimental experience. Additionally, we did not repeat $\mathrm{TH}$ in rhesus macaques due to budget limitations and ethical concerns. In the near future, we will optimize $\mathrm{TH}$ with different onset times of treatment, therapeutic durations, temperature ranges, dosages, and formulae of cold-protective reagents in the goat, and then test the optimized TH strategy in the non-human primate model.

\section{Further application of this model}

In the present study, we safely exposed the chiasmatic and pre-chiasmatic ON within the spacious sphenoid sinus. This allows for several promising avenues of research. (1) Access to the ON allows for other ON disorder models, such as optic neuritis or ischemic optic neuropathy, simply by introducing local damage. (2) Optical and electrical diagnostic tools may be placed in situ, including confocal endomicroscopy ${ }^{42}$ and endoscopic OCT. (3) In situ treatments can be tested at the pre-chiasmatic and chiasmatic ON, to prevent axonal degeneration and promote 
long-distance axonal regeneration by modulating the micro-environment. These treatments could be partnered with intra-vitreal manipulations to maximize RGC survival and ON regeneration. (4) Our work may potentially be applied in other CNS traumas such as SCI or diffused axonal injury in TBI, as well as early axonal degeneration in Alzheimer's, Parkinson's, and normal $\operatorname{aging}^{43}$.

\section{MATERIALS AND METHODS}

\section{Study Design}

This study sought to establish a de novo, clinically-relevant, large animal TON framework, and to develop a novel local TH combined with cold protection. Due to ethical issue and limitations of housing space and other resources, we only used 3-6 goats/rhesus macaques in each group according to our previous experimental experience. At least 3 animals were used per test. For in vivo tests, histology, and transcriptomic studies, sampling and experimental replicates are detailed in the Methods. For ex vivo studies, retinal explants were randomly assigned to three experimental groups $\left(0^{\circ} \mathrm{C}, 0^{\circ} \mathrm{C}\right.$ with $\left.\mathrm{PI}, 37^{\circ} \mathrm{C}\right)$. For in vivo studies, goats from the same company, of the same gender, and of similar ages and weights, were allocated to the groups of injury, hypothermia combined with PI, hypothermia alone, and PI alone without randomization. All the surgeries were performed by one surgeon, and the investigators collecting and analyzing the data were blinded to the grouping. No data outliers were excluded.

\section{Saanen goats and rhesus macaques}

Experiments were conducted following the Association for Research in Vision and Ophthalmology (ARVO) Statement for the Use of Animals in Ophthalmic and Vision Research guidelines. All protocols were approved by the Institutional Animal Care and Use Committee in 
the Wenzhou Medical University (Wenzhou, China, ID number: wydw2020-0789) and the Joinn housed in the animal facility of the Wenzhou Medical University. The rhesus macaques aged animals were housed in an air-conditioned room $\left(21 \pm 2^{\circ} \mathrm{C}\right)$ under a normal light/dark cycle intravenously) after general anesthesia using xylazine $(3 \mathrm{mg} / \mathrm{kg}, \mathrm{IM})$. Rhesus macaques were tiletamine/zolazepam).

\section{Trans-nasal endoscopic procedure to expose the pre-chiasmatic $\mathrm{ON}$ in goats and rhesus}

Goats were anesthetized with xylazine, and then intubated with a $6.0 \mathrm{~mm}$ tracheal tube (Henan oxygen and air (1:1) at a rate of $2 \mathrm{~L} / \mathrm{min}$ by using a mechanical ventilator. Then atropine, ceftiofur sodium, gentamicin, dexamethasone, esmolol hydrochloride and hemocoagulase atrox were injected (detailed information of each drug is listed in the "Drug summary"). vertical double-T-shaped incision on the nose was made through the skin and periosteum.

410 Following blunt dissection, the underlying nasal bone was removed to access the nasal cavity.

411 Under trans-nasal endoscopy (Delong, HD380B), the middle turbinate and posterior olfactory

412 nerve filaments were partially removed by an endoscopic microdebrider (Medtronic, 1884004)

413 powered by the Integrated Power Console system (Medtronic, Integrated Power Console ENT 
414 Surgery, 1898001) to expose the ipsilateral anterior wall of the sphenoid bone. To increase

415 surgical operating space, the nasal septum and part of the contralateral olfactory nerve filaments

416 were also resected to fully expose the anterior bony wall of the sphenoid bone (Figure 1E, left

417 panel). To access the pre-chiasmatic ON within the sphenoid bone, an endoscopic microdrill

418 (Medtronic, diamond microdrill, 1882969) was employed to drill the overlying cortical bone.

419 The posterior myeloid tissue within the sphenoid bone body (Figure 1E, right panel) was

420 aspirated and drilled to create an artificial sphenoid sinus, within which the chiasmatic and pre-

421 chiasmatic ON lay posteriorly (Figure 1F). There are two ways to identify the optic canal (part

422 of the pre-chiasmatic $\mathrm{ON}$ ) in goats during the endoscopic surgery, either by exposing the anterior

423 bony wall of the optic chiasm and then pinpointing the optic canal at the junction of the optic

424 chiasm and the medial orbital wall (Figure 1G) or by using a surgical navigation system.

In the sham surgery group, the ON was exposed by drilling through its anterior bony wall

(Figure 1G). In the ON crush injury group: the ON was crushed by using the blunt blade ( $3 \mathrm{~mm}$ long, $0.5 \mathrm{~mm}$ wide) of a periosteal elevator (HBB010, Shanghai Medical Instruments, China) for 5 seconds (Figure 2A, B).

Following ON injury, the nasal cavity was irrigated consecutively with povidone-iodine and saline, and the artificial sphenoid sinus was filled with an absorbable gelatin sponge (Xiangen Medical Technology, China). After closure of the nasal periosteum and the skin with 3-0 sutures, anesthesia recovery was conducted.

The trans-nasal endoscopic procedure to expose the optic canal in rhesus macaque was similar to that in goats and used the same surgical equipment. Our self-designed surgical path planning program helped screen for suitable macaques and determine the surgical path based on their presurgical cranial CT scans (Supplementary Movie 9). The rhesus macaque was anesthetized by 
437 isoflurane (1.5-2\%) after induction by Zoletil50 (4-8 mg/kg IM, Virbac, France) in the supine

438 position. The exposed pre-chiasmatic ON was confirmed by a surgical navigation system (NDI

439 Polaris Vicra).

440 Computer-assisted semi-automated surgical path planning in trans-nasal endoscopic surgery based on CT scans

442 To facilitate endoscopic exposure and expand this surgical approach to other large animal

443 species, a computer-assisted surgical path planning program was designed based on pre-surgical

444 cranial CT scans. The flowchart of the program is shown in Figure 6A. The major steps were as

445 follows:

446 (1) Virtual reconstruction of surgical space based on cranial CT scans. Cranial CT scans with

447 slice thicknesses of $0.65 \mathrm{~mm}$ in goats and $1 \mathrm{~mm}$ in beagles and macaques were obtained before

448 surgery (pigs and goats, GE Optina660, General Electric Company, Boston, USA; beagles and

449 macaques, Philip Brilliance iCT, Royal Philips Company, Amsterdam, Holland). The surgical

450 space was manually segmented in the MITK software (MITK, v2018.04.2, detailed code is

451 available in http://mitk.org/wiki/Downloads). The anatomic boundaries of the surgical space

452 were as follows: the nasal and maxillary bone anteriorly, the anterior skull base superiorly, the

453 palate inferiorly, the medial orbital walls laterally, and the posterior wall of the sphenoid body

454 posteriorly (Figure 6B, in green). The anterior wall of the left optic canal was marked as the

455 surgical target (Figure 6B, in yellow) that needed to be approached by the surgical microdrill.

456 (2) Virtual reconstruction of the surgical microdrill and the surgical corridor (formed by the 457 movement of the surgical microdrill along its major axis) (Figure 6C). 
(3) Calculation and optimization of the surgical path. There were five degrees of freedom for the movement of the surgical corridor when its tip met the optic canal (Figure 6D). Using a comprehensive search in the program, all possible surgical paths in the three-dimensional virtual space were tested and screened for the most feasible paths in which all the pixels within the surgical corridor were either in the confined surgical space or in air (space outside of the animal's skull) (Figure 6E).

(4) Optimization of microdrill size. If no feasible surgical path was identified, the computer automatically decreased the diameter of the surgical microdrill by $0.5 \mathrm{~mm}$, and then repeated steps (1) - (3) to screen for feasible paths.

The code of the computer program is available online (https://github.com/LujieZhang/Preoperative-planning).

\section{Evaluation of microtubule cold-stability in the retinal explants of goats}

Goat eyecups were prepared within 15 mins after euthanization with $10 \%$ potassium chloride (40 $\mathrm{ml}$, intravenously) in conjunction with general anesthesia using xylazine. Three neighboring retinal pieces, $2 \mathrm{~mm}$ in diameter, were taken from the peripheral retina, and randomly assigned to three experimental groups $\left(0^{\circ} \mathrm{C}, 0^{\circ} \mathrm{C}\right.$ with PI (cold-protective reagent), $\left.37^{\circ} \mathrm{C}\right)($ Figure $5 \mathbf{A})$. The retinal explants were pre-treated in hibernate A medium (BrainBits, 2283833, USA) with or without PI (1:500, EMD Millipore Company, 539134, Germany) for 30 minutes at room temperature, then were kept in a $37^{\circ} \mathrm{C}$ incubator or in a $0^{\circ} \mathrm{C}$ ice-water mixture for 6 hours. After fixation in 4\% PFA for 30 minutes, the retinal explants were immuno-stained against microtubule with beta-3 tubulin (TUBB3) antibody (1:250, from rabbit, Cell Signaling Technology, 5568S, USA), washed in $0.5 \%$ Triton in PBS for 5 times, and then were immunestained with Alexa Fluor 488 conjugated anti-rabbit secondary antibody (1:200, from donkey, 
Thermo Fisher Scentific, A-21206, USA). Confocal images were taken under confocal microscopy (Cell Observer SD, ZEISS, Germany) with 63X oil objective lens. Accumulative axonal length of TUBB3-positive optic nerve was analyzed using computer-assisted software ('Simple Neurite Tracer' plugin, ImageJ). Axonal beads along the TUBB3-positive optic nerve were counted manually and bead density was calculated as the number of beads per $100 \mu \mathrm{m}$ TUBB3-positive optic nerve. For each retinal explant, three regions (108.36 $\mu \mathrm{m}$ x $108.36 \mu \mathrm{m}$, 512 x 512 pixels) were imaged, and the accumulative axonal length and axonal bead densities in these three regions were averaged to yield one readout. Retinal pieces with many optic nerve bundles were excluded to facilitate precise quantification. 11 retinal explants from 4 male goats were used in each experimental group. After testing for normality, a repeated measures one-way ANOVA was applied with Dunnett's multiple comparisons test.

\section{Trans-nasal local hypothermic therapy}

We developed a trans-nasal local cooling system. Cooling water was pumped into a nasal irrigation tube by a rotary pump (Beijing Zhongshidichuang Technology Development Co., Ltd, China). An irrigation and drainage system consisted of a double-tube cooling device including a pair of concentric silicon tubes. The inner silicon tube (irrigation tube, $3 \mathrm{~mm}$ in diameter, Zhejiang Kangkang Medical Equipment Co., Ltd, China) was connected to the pump to receive the cool water. The outer silicon tube (drainage tube, $7 \mathrm{~mm}$ in diameter, Taizhou Biling Hardware Products Co., Ltd., China) was covered with cling film (Shanghai Runwen Packaging Materials Co., Ltd., China) to drain the water out of the nasal cavity (Figure 5E). A gelfoam sponge (Xiang'en Jiangxi Medical Technology Development Co., Ltd., China) filled with goat serum (Beyotime Institute of Biotechnology, C0265, China) with or without 1:200 diluted PI (EMD Millipore Company, 539134, Germany) was placed between the injured ON and the cling 
504 film (Figure 5E, F). To test the effect of hypothermia and PI (cold-protective reagents) on

505

506

507

508

509

510

511

512

513

514

515

516

517

518

519

520

521

522

injured ONs, goats were assigned to the following three experimental groups: (1) local

hypothermia with PI, (2) local hypothermia without PI, and (3) PI without local hypothermia.

Immediately after the $\mathrm{ON}$ crush injury, the sponge was placed at the injury site and then the local cooling tubes were inserted over the gelfoam sponge (Supplementary Movie 4). The iced water mixture $\left(0^{\circ} \mathrm{C}\right.$ as measured by a digital thermometer; ZS-T, Beijing Zhongshidichuang Technology Development Co., Ltd., China) was pumped into the double-tube device to cool the outer surface of the cling film to $4^{\circ} \mathrm{C}$ (measured by the same digital thermometer). After 2 hours of continuous cooling, the iced water mixture was replaced consecutively with water at 5, 10, 15, and $20^{\circ} \mathrm{C}$ water for $15 \mathrm{~min}$ each to achieve slow rewarming (Figure 5G). Then the goat was awakened from general anesthesia. Room temperature was maintained at $23^{\circ} \mathrm{C}$.

\section{RNA-sequencing}

Fresh samples from the goat's retina, retrobulbar, and pre-chiasmatic ON in injury and contralateral eyes were harvested and frozen in liquid nitrogen within 30 minutes after euthanization at 1 dpi (Figure 4A). RNA extraction and sequencing was completed in a biocompany (Biomarker Technologies, China). Total RNA of each sample was extracted following the instruction manual of the TRIzol Reagent (Life Technologies, CA, USA). Agilent 2100 Bioanalyzer (Agilent Technologies, Inc., Santa Clara, CA, USA) was used to test the RNA integrity and concentration. The mRNA was isolated by NEBNext Poly (A) mRNA Magnetic Isolation Module (NEB, E7490), then fragmented into approximately $200 \mathrm{nt}$ RNA inserts. The cDNA library was constructed according to the NEBNext Ultra RNA Library Prep Kit for Illumina (NEB, E7530) and NEBNext Multiplex Oligos for Illumina (NEB, E750), and was sequenced using an Illumina HiSeq ${ }^{\mathrm{TM}}$ sequencing platform. The reads were mapped to ARS1 
527 (Capra hicus) genome (GenBank assembly accession: GCA_001704415.1) using Tophat2

528 software. Gene expression levels were estimated using Log2 CPM transformation values on

529 NetworkAnalyst 3.0 website. The raw base call (.bcl) files were converted to demultiplexed

$530 \quad$ FASTQ files with Bcl2fastq v2.19.1 for data analysis. The RNA-sequencing and analysis were

531 repeated in 4 goats. The RNA-seq data has been uploaded online

532 (https://www.ncbi.nlm.nih.gov/geo/query/acc.cgi?\&acc=GSE182164).

RNA-seq analysis

Raw reads were assessed using the FastQC toolset

535 (http://www.bioinformatics.babraham.ac.uk/projects/fastqc). The statistics on the raw reads and

536 alignments are shown in sheet 'Mapping Statistics', Data S3. R Subread package was used to

537 quantify gene expression. Adjusted p values from differential expression tests were calculated

538 using the Benjamini-Hochberg procedure for multiple hypothesis testing. Gene ontology (GO)

539 analysis was performed based on hypergeometric test using clusterProfiler package.

540 To obtain the downstream protein-protein interaction map, the differentially expressed genes

541 were analyzed in the STRING database (https://string-db.org/). Genes that interact more with

542 other genes at the protein level were analyzed in the Cytoscape software (version 3.8.2). The

543 genes with high connectivity were named as hub genes.

\section{Flash visual evoked potential (FVEP)}

545 Visual evoked potential (FVEP) is the cortical potential in response to visual stimulus, reflecting

546 the function of retino-geniculate pathway ${ }^{19}$. FVEP recording in goats has been previously

547 reported in detail ${ }^{24}$. Briefly, following general anesthesia and electrode placement, the animal

548 was adapted to the testing environment for 5 minutes. Then the FVEP was recorded at intensities 
of 0.025 and $0.25 \mathrm{~cd} \cdot \mathrm{s} / \mathrm{m}^{2}$ consecutively (GT-2008V-III, GOTEC Co., Ltd, China). To reduce variation, we used the FVEP amplitude ratio of the surgical eye to the contralateral eye to quantify the FVEP changes in the surgical eyes.

The pattern visual evoked potential (PVEP) test in rhesus macaques was described previously ${ }^{24}$. The PVEP, which is more reproducible than FVEP in human ${ }^{19}$, can be elicited in rhesus macaques according to our previous study ${ }^{24}$. Therefore, we replaced the FVEP test with the PVEP test in rhesus macaques.

\section{Pattern electroretinogram (PERG) recordings}

PERG is the retinal electrical signal activated by patterned visual stimuli, the amplitude of which indicates the functional integrity of $\mathrm{RGCs}^{20}$. PERG recording in goats and rhesus macaques has been previously reported ${ }^{24}$. Briefly, the goat was anesthetized with xylazine. After electrode placement, PERG signals were elicited by contrast-reversal black-white checkerboards (temporal frequency, $2.4 \mathrm{~Hz}$ ) at spatial frequencies of $0.1,0.3,1.0,3.0$ per degree consecutively (GT2008V-III, GOTEC Co., Ltd, China). To reduce variation, we report a ratio of the PERG amplitude of the surgical eye to the contralateral eye.

\section{Pupillary light response (PLR) test}

The PLR test was used to evaluate the integrity of the ocular afferent (ON) and the efferent (oculomotor nerve) pathways ${ }^{18}$. Direct PLR (dPLR) is the PLR in the ipsilateral eye while indirect PLR (iPLR) occurs in the contralateral eye. This method has been previously described $^{24}$. Briefly, the goat was dark-adapted for 5 minutes after anesthesia with xylazine. Two infrared cameras were positioned at $5 \mathrm{~cm}$ from each eye and focused on the pupillary plane to film the PLR. Pupillary constriction was elicited by white light stimulus (230 lx) for two 
seconds. The PLR results were scored manually by the same person as follows: Grade 2 (score $=$ 2) $=$ normal PLR; Grade $1($ score $=1)=$ delayed or weak PLR; Grade $0($ score $=0)=$ no PLR.

\section{Spectral-domain optical coherence tomography (SD-OCT) imaging}

Retinal OCT imaging was used to measure the thickness of the retinal ganglion cell complex including the RNFL, RGC and IPL layers, which represent the RGC axons, somas and dendrites, respectively. This method has been previously described ${ }^{24}$. Briefly, after anesthesia, the OCT images were taken with peripapillary circular scan pattern (Heidelberg Spectralis OCT system, Germany). The thickness of the GCC was measured manually using the Heidelberg software.

\section{Immunohistochemical (IHC) staining of retinal ganglion cells (RGCs)}

The IHC staining for RGCs was performed as described in our previous study ${ }^{24}$. Briefly, the eyecup was made and fixed in 4\% paraformaldehyde in phosphate-buffered saline (PBS) for $24 \mathrm{~h}$ at $4^{\circ} \mathrm{C}$. For each retinal quadrant, three circular retinal pieces with a radius of $1 \mathrm{~mm}$ were dissected out using a corneal trephine blade (Zhonglindongsheng Medical Instrument, Jiangsu, China) at the central, mid-peripheral and peripheral retina $(1 / 4,1 / 2$ and $3 / 4$ of the retinal radius away from the $\mathrm{ON}$ head, respectively). For each eye, the RGC densities of 12 retinal pieces were averaged to yield one readout. Retinal samples were blocked in 5\% goat serum (C0265, Beyotime Institute of Biotechnology, China) in 0.5\% PBST overnight, and then incubated in a 1:4000 diluted primary antibody (anti-RBPMS from guinea pig, ProSci, California, custommade) in $0.5 \%$ PBST for $24 \mathrm{~h}$ at room temperature. After being washed in PBS, the retinal pieces were incubated in a 1:200 diluted secondary antibody in PBS (Cy3-conjugated, anti-guinea pig from goat, Jackson Immuno Research, West Grove, Pennsylvania) for $12 \mathrm{~h}$ at room temperature. The retinal pieces were then washed before being mounted on slides. Confocal images were taken using a Zeiss LSM710 system (Carl Zeiss Meditec, Sartrouville, Germany) under a 20X 
594 objective lens. RBPMS-positive RGCs were counted manually using ImageJ software (NIH, Bethesda, MD, USA).

597 Dissection of the ON was completed within 30 mins after euthanization. The ON was crosssectioned into 1-2 mm-thick discs at the retrobulbar (2 $\mathrm{mm}$ behind the eyeball, named as \#1), mid-orbital (\#2), intra-canalicular (lesion site, \#3) and pre-chiasmatic (post-injury site, \#4) regions. The ON sections were fixed in $2 \%$ glutaraldehyde and $2 \% \mathrm{PFA}$ in PBS for $24 \mathrm{~h}$ at $4{ }^{\circ} \mathrm{C}$. After being washed in $0.1 \mathrm{M} \mathrm{PB}$, the samples were incubated in $1 \%$ osmium tetroxide in $0.1 \mathrm{M}$ PBS for $1 \mathrm{~h}$ and then incubated in $2 \%$ uranyl acetate in double distilled water for $1.5 \mathrm{~h}$ at $37^{\circ} \mathrm{C}$. The samples were then dehydrated through a series of graded ethanol (50\% to $100 \%$ in $\mathrm{H} 2 \mathrm{O})$ for $10 \mathrm{~min}$ at each concentration. The samples were then embedded in 50\% EMbed 812/50\% propanone for $1 \mathrm{~h}$, followed by a 4:1 ratio of EMbed 812/propanone overnight at $37^{\circ} \mathrm{C}$. The next day, the samples were transferred to $100 \%$ EMbed 812 for $1 \mathrm{~h}$ at $45^{\circ} \mathrm{C}$ and embedded in a mold filled with $100 \%$ EMbed 812 at $45^{\circ} \mathrm{C}$ for $3 \mathrm{~h}$ and then at $65^{\circ} \mathrm{C}$ for 2 days. Semi-thin sections (2 $\mu \mathrm{m})$ were cut using an ultramicrotome (LKB-2088, LKB, Bromma, Sweden), stained with $1 \%$ para-phenylenediamine (PPD) in methanol: isopropanol (1:1) for $35 \mathrm{~min}$ and then rinsed three times with methanol:isopropanol (1:1). Myelin and unhealthy axonal cytoplasm were stained with PPD. More than five separate regions $(125.22 \mu \mathrm{m}$ x $94.26 \mu \mathrm{m})$ of each section were imaged at 70X magnification using a Leica DM4B epifluorescence microscope. The surviving axons were semi-automatically counted to obtain the average surviving axonal density for each section.

614 Since the axonal densities amongst different ON sites of the contralateral eyes were statistically

615 the same at 1 and 3 mpi after ON crush (Supplementary Figure 3C), we used the axonal density 616 at region \#3 to represent the axonal density in the contralateral eye. 


\section{Materials \& Correspondence}

618 Correspondence and material requests should be addressed to Yikui Zhang, 86-13705770161,

619 zhang.yikui@wmu.edu.cn.

\section{0 \\ Data and materials availability}

621 Computer program download site:

622 https://github.com/LujieZhang/Preoperative-planning.

623 The processed gene expression data in this paper have been deposited into the NCBI GEO

624 database: GSE182164. RNA-seq data download site:

625 https://www.ncbi.nlm.nih.gov/geo/query/acc.cgi?\&acc=GSE182164.

\section{Statistical analyses}

627 All data were analyzed using GraphPad (8.0) software. Normality tests were used to analyze the 628 distributions of all data sets. To compare two groups of data, Student's t-test or nonparametric 629 test was used. To compare multiple groups, a one-way ANOVA (with Dunnett's multiple630 comparisons test) or nonparametric Kruskal-Wallis test (with Dunn's multiple-comparisons test) 631 was used. Two-way ANOVA (with multiple comparisons) was used to analyze OCT, PERG, 632 FVEP, PVEP and axonal density data. Asterisks $\left(^{*}\right)$ represent statistically significant differences $633(* \mathrm{p}<0.05, * * \mathrm{p}<0.01, * * * \mathrm{p}<0.001, * * * * \mathrm{p}<0.0001)$. Data are presented as mean \pm s.e.m.

\section{Author contributions:}

635 Conceptualization: YZ, WL, JY, YH, WW

636 Methodology: YZ, ML, BY, SL, LZ, SZ, HH, QY, JS, WJ, HZ, PH, ZY, TX, WL, DA, JF, HS, 637 YJ, XL, XC, JO, WL, LS, YSS 
638 Investigation: YZ, ML, BY, SL, LZ, SZ, HH, QY, JS, WJ, HZ, PH, ZY, TX, WL, DA, JF, HS,

639 YJ, XL, XC, JO, WL, LS

640 Visualization: YZ, ML, SL,

641 Funding acquisition: WW, YZ, JY

642 Project administration: WW, YZ

643 Supervision: YZ, WW

644 Writing - original draft: YZ

645 Writing - review \& editing: YZ, JO, WL, YH, WW 


\section{Acknowledgments:}

647 We thank Dr. Haohua Qian for critically reading this manuscript. We thank Dr. Michael W.

648 Country for editing this manuscript. We also appreciate Professor Kaihui Nan, Yu Xia, Yuanfei

649 Ji, Mingna Xu, Qiqi Xie, Weijie Liu, Zhaoqi Pan, Xiaohui Jiang, Yao Zhou, Mengting Jin,

650 Haochen Jin for taking part in experimental conduction.

\section{Funding:}

652 National Key R\&D Program of China (2016YFC1101200)

653 National Natural Science Foundation of China (81770926;81800842)

654 Key R\&D Program of Zhejiang Province (2018C03G2090634)

655 Key R\&D Program of Wenzhou Eye Hospital (YNZD1201902)

656 National Key R\&D Program of China (2019YFC0119300)

657 The sponsor or funding organization had no role in the design or conduct of this research. 


\section{Reference}

1. Wang, H., Olivero, W., Wang, D. \& Lanzino, G. Cold as a therapeutic agent. Acta Neurochir (Wien) 148, 565-570 (2006).

2. Martirosyan, N.L., et al. The role of therapeutic hypothermia in the management of acute spinal cord injury. Clin Neurol Neurosurg 154, 79-88 (2017).

3. Clifton, G.L., et al. Lack of effect of induction of hypothermia after acute brain injury. $N$ Engl J Med 344, 556-563 (2001).

4. Clifton, G.L., et al. Very early hypothermia induction in patients with severe brain injury (the National Acute Brain Injury Study: Hypothermia II): a randomised trial. The Lancet. Neurology 10, 131-139 (2011).

5. Kramer, C., et al. Therapeutic hypothermia for severe traumatic brain injury: a critically appraised topic. The neurologist 18, 173-177 (2012).

6. Schubert, A. Side effects of mild hypothermia. Journal of neurosurgical anesthesiology 7, 139-147 (1995).

7. Ou, J., et al. iPSCs from a Hibernator Provide a Platform for Studying Cold Adaptation and Its Potential Medical Applications. Cell 173, 851-863.e816 (2018).

8. Chamoun, R. \& Couldwell, W.T. Practical and technical aspects of trans-sphenoidal surgery. J Neurosurg Sci 55, 265-275 (2011).

9. Casler, J.D., Doolittle, A.M. \& Mair, E.A. Endoscopic surgery of the anterior skull base. Laryngoscope 115, 16-24 (2005).

10. Goudakos, J.K., Markou, K.D. \& Georgalas, C. Endoscopic versus microscopic trans-sphenoidal pituitary surgery: a systematic review and meta-analysis. Clin Otolaryngol 36, 212-220 (2011).

11. Yan, W., et al. Incidence of optic canal fracture in the traumatic optic neuropathy and its effect on the visual outcome. The British journal of ophthalmology 101, 261-267 (2017).

12. Yu-Wai-Man, P. \& Griffiths, P.G. Steroids for traumatic optic neuropathy. The Cochrane database of systematic reviews, CD006032-CD006032 (2013).

13. Kalds, P., et al. Sheep and Goat Genome Engineering: From Random Transgenesis to the CRISPR Era. Frontiers in Genetics 10(2019).

14. Araújo, A.M.d., et al. Genetic diversity between herds of Alpine and Saanen dairy goats and the naturalized Brazilian Moxotó breed. Genetics and Molecular Biology 29, 67-74 (2006).

15. Martin, P., et al. Genome-wide association mapping for type and mammary health traits in French dairy goats identifies a pleiotropic region on chromosome 19 in the Saanen breed. Journal of Dairy Science 101, 5214-5226 (2018).

16. Gipson, T.A. Recent advances in breeding and genetics for dairy goats. Asian-Australas J Anim Sci 32, 1275-1283 (2019).

17. Yu-Wai-Man, P. Traumatic optic neuropathy-Clinical features and management issues. Taiwan journal of ophthalmology 5, 3-8 (2015).

18. Kerrison, J.B., et al. Quantification of optic nerve axon loss associated with a relative afferent pupillary defect in the monkey. Arch Ophthalmol 119, 1333-1341 (2001).

19. Odom, J.V., et al. ISCEV standard for clinical visual evoked potentials (2009 update). Documenta ophthalmologica. Advances in ophthalmology 120, 111-119 (2010).

20. Porciatti, V. Electrophysiological assessment of retinal ganglion cell function. Exp Eye Res 141, 164-170 (2015).

21. Singman, E.L., et al. Indirect traumatic optic neuropathy. Military Medical Research 3, 2-2 (2016).

22. Miyahara, T., Kurimoto, Y., Kurokawa, T., Kuroda, T. \& Yoshimura, N. Alterations in retinal nerve fiber layer thickness following indirect traumatic optic neuropathy detected by nerve fiber analyzer, GDx-N. Am J Ophthalmol 136, 361-364 (2003).

23. Shi, W., et al. Axonal loss and blood flow disturbances in the natural course of indirect traumatic optic neuropathy. Chin Med J (Engl) 126, 1292-1297 (2013).

24. Zhang, Y., et al. In vivo evaluation of retinal ganglion cells and optic nerve's integrity in large animals by multi-modality analysis. Exp Eye Res 197, 108117 (2020).

25. Richardson, P.M., Issa, V.M. \& Shemie, S. Regeneration and retrograde degeneration of axons in the rat optic nerve. J Neurocytol 11, 949-966 (1982).

26. TM., P. Specializations of the human visual system: the monkey model meets human reality. (ed. Kaas JH, C.C.) 2004:2231-2059 (Boca Raton, FL: CRC Press, 2004). 
27. Levin, L.A., Beck, R.W., Joseph, M.P., Seiff, S. \& Kraker, R. The treatment of traumatic optic neuropathy: the International Optic Nerve Trauma Study. Ophthalmology 106, 1268-1277 (1999).

28. TH, H. \& S, D.G. The translational landscape in spinal cord injury: focus on neuroplasticity and regeneration. Nature reviews. Neurology 15, 732-745 (2019).

29. Aguayo, A.J., et al. Degenerative and regenerative responses of injured neurons in the central nervous system of adult mammals. Philosophical transactions of the Royal Society of London. Series B, Biological sciences 331, 337-343 (1991).

30. Upadhyay, R.K. Drug delivery systems, CNS protection, and the blood brain barrier. Biomed Res Int 2014, 869269-869269 (2014).

31. Coles, J.P. Regional ischemia after head injury. Current opinion in critical care 10, 120-125 (2004).

32. Thale, A., Jungmann, K. \& Paulsen, F. Morphological studies of the optic canal. Orbit 21, 131-137 (2002).

33. Dietrich, W.D. \& Bramlett, H.M. Therapeutic hypothermia and targeted temperature management for traumatic brain injury: Experimental and clinical experience. Brain Circ 3, 186-198 (2017).

34. Evanson, N.K., Guilhaume-Correa, F., Herman, J.P. \& Goodman, M.D. Optic tract injury after closed head traumatic brain injury in mice: A model of indirect traumatic optic neuropathy. PLoS One 13, e0197346 (2018).

35. Tao, W., et al. A Novel Mouse Model of Traumatic Optic Neuropathy Using External Ultrasound Energy to Achieve Focal, Indirect Optic Nerve Injury. Sci Rep 7, 11779 (2017).

36. Bastakis, G.G., Ktena, N., Karagogeos, D. \& Savvaki, M. Models and treatments for traumatic optic neuropathy and demyelinating optic neuritis. Developmental Neurobiology 79, 819-836 (2019).

37. Friedli, L., et al. Pronounced species divergence in corticospinal tract reorganization and functional recovery after lateralized spinal cord injury favors primates. Sci Transl Med 7, 302ra134 (2015).

38. Nardone, R., et al. Rodent, large animal and non-human primate models of spinal cord injury. Zoology (Jena) 123, 101-114 (2017).

39. Zhang, Y., et al. In vivo evaluation of outer retinal function and structure after retrobulbar optic nerve crush by lateral orbitotomy in goats. Exp Eye Res 209, 108652 (2021).

40. Mesentier-Louro, L.A., et al. Direct targeting of the mouse optic nerve for therapeutic delivery. Journal of Neuroscience Methods 313, 1-5 (2019).

41. Yang, S.-G., et al. Strategies to Promote Long-Distance Optic Nerve Regeneration. Frontiers in Cellular Neuroscience 14(2020).

42. Belykh, E., et al. Progress in Confocal Laser Endomicroscopy for Neurosurgery and Technical Nuances for Brain Tumor Imaging With Fluorescein. Front Oncol 9, 554 (2019).

43. Salvadores, N., Sanhueza, M., Manque, P. \& Court, F.A. Axonal Degeneration during Aging and Its Functional Role in Neurodegenerative Disorders. Frontiers in neuroscience 11, 451-451 (2017). 
Table 1. Drug summary

\begin{tabular}{|c|c|c|c|}
\hline Drug & Function & $\begin{array}{l}\text { Dosage, } \\
\text { delivery }\end{array}$ & Source \\
\hline Xylazine & General anesthesia & $\begin{array}{l}4 \mathrm{mg} / \mathrm{kg}, \\
\text { intramuscular } \\
\text { (IM) }\end{array}$ & $\begin{array}{l}\text { Huamu Animal } \\
\text { Health Products, } \\
\text { China }\end{array}$ \\
\hline Idzoxan & Antagonist of xylazine & $1.5 \mathrm{mg} / \mathrm{kg}, \mathrm{IM}$ & $\begin{array}{l}\text { Huamu Animal } \\
\text { Health Products, } \\
\text { China }\end{array}$ \\
\hline Propofol & $\begin{array}{l}\text { Induction of general } \\
\text { anesthesia }\end{array}$ & $\begin{array}{l}5 \mathrm{mg} / \mathrm{kg} \text {, } \\
\text { intravenous } \\
\text { (IV) }\end{array}$ & $\begin{array}{l}\text { Xian Lipont } \\
\text { Enterprise Union } \\
\text { Management, China }\end{array}$ \\
\hline Isoflurane & General anesthesia & $\begin{array}{l}2.5-3.5 \% \text {, by } \\
\text { mechanical } \\
\text { ventilator }\end{array}$ & $\begin{array}{l}\text { RWD Life Science, } \\
\text { China }\end{array}$ \\
\hline Atropine & $\begin{array}{l}\text { Reduction of bronchial } \\
\text { secretion, cardiac } \\
\text { protection }\end{array}$ & $0.05 \mathrm{mg} / \mathrm{kg}, \mathrm{IV}$ & $\begin{array}{l}\text { Guangdong Jieyang } \\
\text { Longyang Animal } \\
\text { Pharmaceutical, } \\
\text { China }\end{array}$ \\
\hline $\begin{array}{l}\text { Esmolol } \\
\text { hydrochloride }\end{array}$ & $\begin{array}{l}\text { Reduction of blood } \\
\text { pressure }\end{array}$ & $\begin{array}{l}0.2 \mathrm{~g}(2 \mathrm{ml}) \text { in } \\
48 \mathrm{ml} \text { saline, }\end{array}$ & $\begin{array}{l}\text { Qilu Pharmaceutical, } \\
\text { China }\end{array}$ \\
\hline
\end{tabular}




\begin{tabular}{|c|c|c|c|}
\hline & & $\begin{array}{l}\text { IV drip } \\
\text { infusion }\end{array}$ & \\
\hline $\begin{array}{l}\text { Hemocoagulase } \\
\text { Atrox }\end{array}$ & $\begin{array}{l}\text { Reduction of } \\
\text { hemorrhage }\end{array}$ & $\begin{array}{l}1 \text { unit (1 shot), } \\
\text { IM }\end{array}$ & $\begin{array}{l}\text { Penglainuokang } \\
\text { Pharmaceutical, } \\
\text { China }\end{array}$ \\
\hline Dexamethasone & Anti-inflammation & $5 \mathrm{ml}(5 \mathrm{mg}), \mathrm{IV}$ & $\begin{array}{l}\text { Kelong Veterinary } \\
\text { Medicine, China }\end{array}$ \\
\hline $\begin{array}{l}\text { Ceftiofur } \\
\text { sodium }\end{array}$ & $\begin{array}{l}\text { Prevention of bacterial } \\
\text { infection }\end{array}$ & $20 \mathrm{mg} / \mathrm{kg}, \mathrm{IM}$ & Jiangxi Huatu, China \\
\hline Gentamicin & $\begin{array}{l}\text { Prevention of bacterial } \\
\text { infection }\end{array}$ & $8 \mathrm{mg} / \mathrm{kg}, \mathrm{IM}$ & $\begin{array}{l}\text { Shanxi Ruicheng } \\
\text { Kelong Co., Ltd, } \\
\text { China }\end{array}$ \\
\hline
\end{tabular}


bioRxiv preprint doi: https://doi.org/10.1101/2021.11.07.467626; this version posted November 8, 2021. The copyright holder for this preprint (which was not certified by peer review) is the author/funder, who has granted bioRxiv a license to display the preprint in perpetuity. It is made available under aCC-BY 4.0 International license.

A

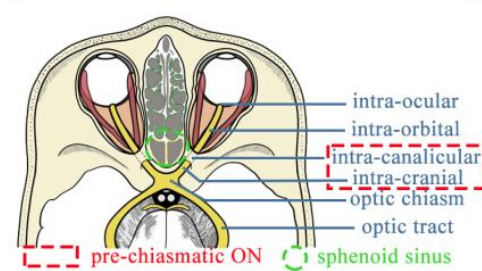

$\mathrm{D}$

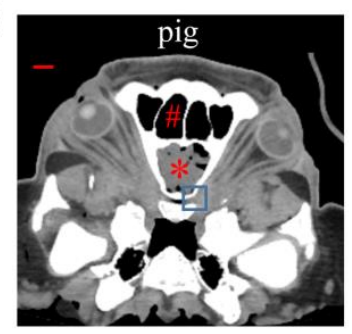

E

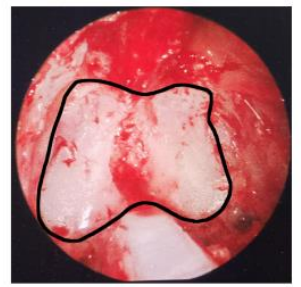

$\square$ cortical bone
$\mathrm{B}$

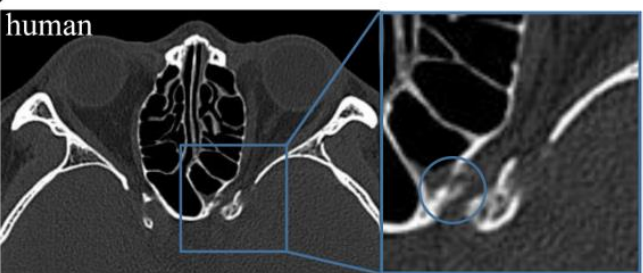

C

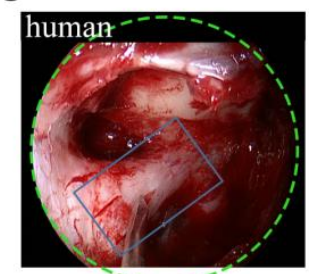

isphenoid sinus $\square$ pre-chiasmatic 0

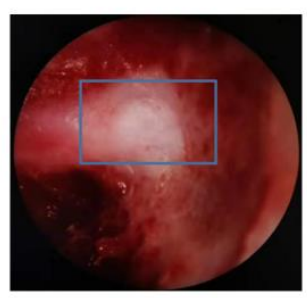

$\square$ pre-chiasmatic ON

$\mathrm{H}$
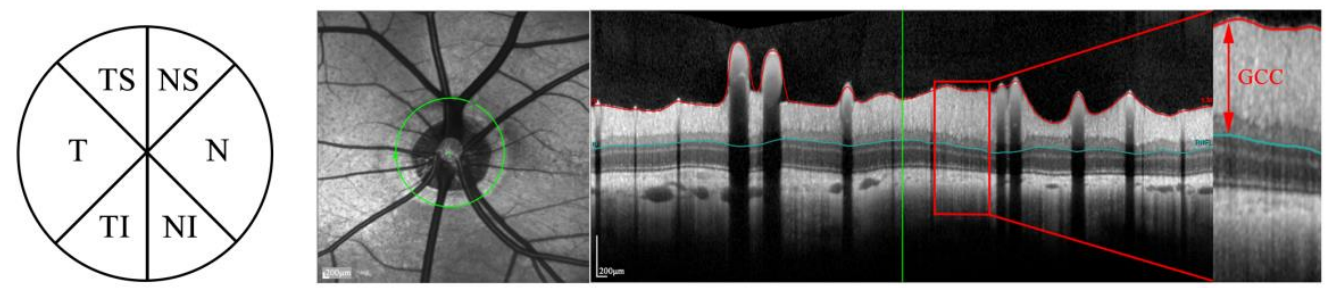

I

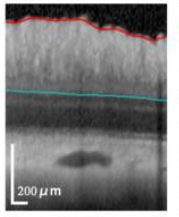

baseline

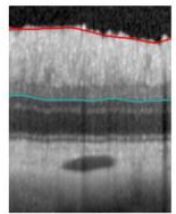

$1 \mathrm{mps}$

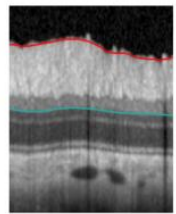

$2 \mathrm{mps}$

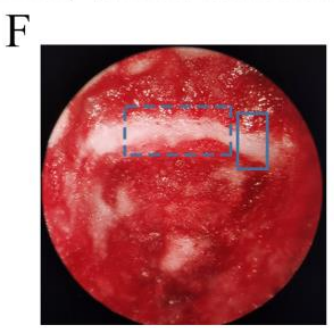

I-joptic chiasm

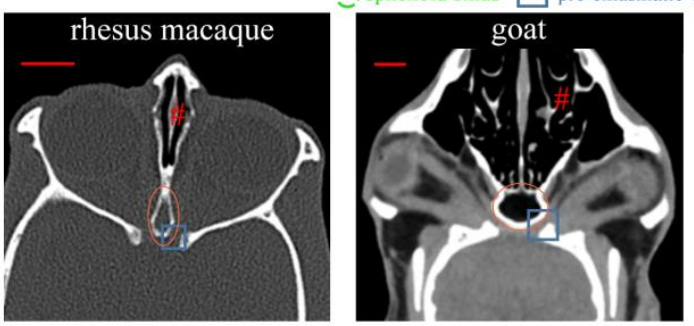

G

G

$\mathrm{J}$

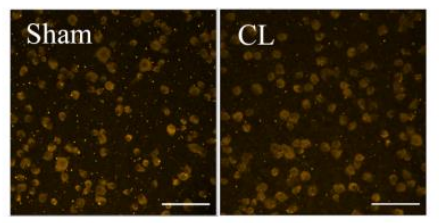

RGC density

$\mathrm{K}$
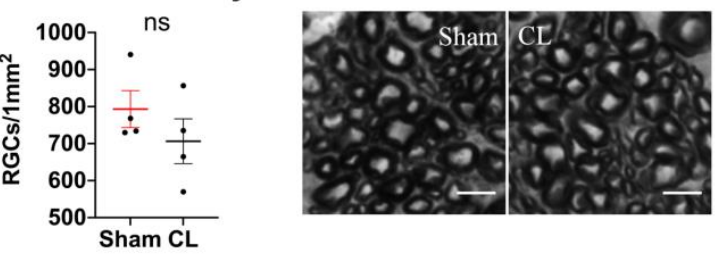

ON density

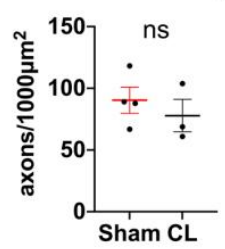




\section{Fig. 1. Trans-nasal endoscopic-mediated exposure of pre-chiasmatic $\mathrm{ON}$ in goat is feasible} and safe. (A) Scheme of anatomic segments of ON and sphenoid sinus (green circle) in human. The pre-chiasmatic ON was shown in the dashed box. (B) CT scan of TON patient with optic canal fracture (blue circle). (C) Endoscopic view of human's prechiasmatic ON (blue rectangle) within the large sphenoid sinus (shown in the green circle). (D) Representative skull CT scans of pre-chiasmatic ON (blue rectangle) in pigs, beagles, rhesus macaques and goats. Number sign indicated the nasal cavity. Asterisk indicated the dropping frontal cortex in pigs and beagles. Red circle indicates the sphenoid bone body in beagles, rhesus macaques and goats. Scale bar $=10 \mathrm{~mm}$. $(\mathrm{E})$ Endoscopic image of a goat's sphenoid bone body, which is made of cortical bone (left panel) and trabecular bone with myeloid tissue (right panel). (F) Endoscopic image of a goat's chiasmatic ON (blue dashed rectangle) and pre-chiasmatic ON (blue rectangle) with bony wall unremoved. (G) Endoscopic exposure of a goat's pre-chiasmatic ON (blue rectangle) with its anterior bony wall removed. (H) Illustration of GCC thickness measurement by OCT retinal imaging around the optic nerve head in six regions $(\mathrm{T}$ : temple, N: nasal; S: superior; I: inferior). (I) Representative OCT images of the eye with its pre-chiasmatic ON exposed (sham surgery eye) (left panel) and quantification of GCC thickness ratio of the sham surgical eyes to the contralateral naïve eyes before and after pre-chiasmatic ON exposure. $n=4$. Two-way ANOVA with Tukey's multiple comparison (compared with the baseline). (J) Representative immunostaining images of the RBMPS positive RGCs in the retinal flat-mounts (left panel) and quantification of RGCs densities of the sham surgical eyes and the contralateral eyes at 3 months after pre-chiasmatic ON exposure (right panel). $\mathrm{n}=4$. Wilcoxon test. Scale bar $=100 \mu \mathrm{m}$. (K) Representative 
microscopic images of semi-thin cross sections of ON (left panel) and quantification of axonal densities of the sham surgical eyes and the contralateral eyes at 3 months after pre-chiasmatic ON exposure (right panel). $n=3-4$. Unpaired t-test. Scale bar $=5 \mu \mathrm{m}$. Data were presented as mean \pm s.e.m. ns: $p>0.05$, not significant. CL: contralateral, mps: month post-surgery (post sham surgery). 
A

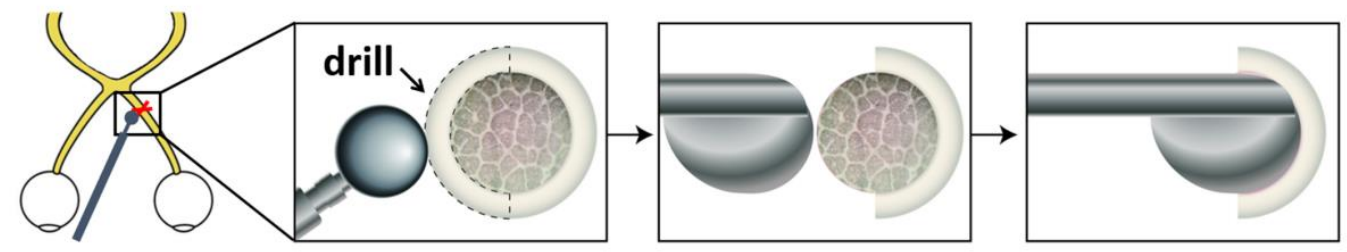

B

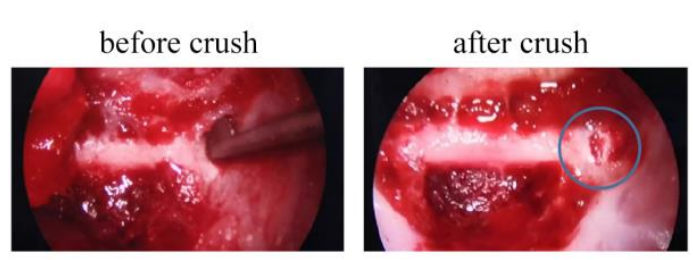

$\mathrm{C}$ Inj dPLR

CL iPLR
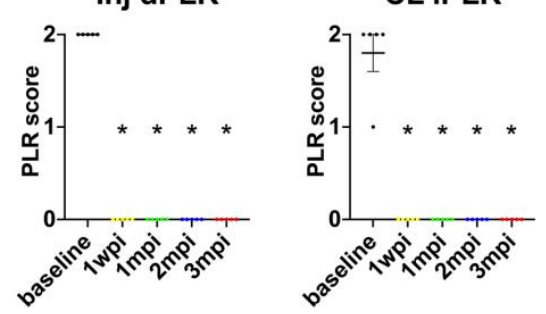

$\mathrm{D}$

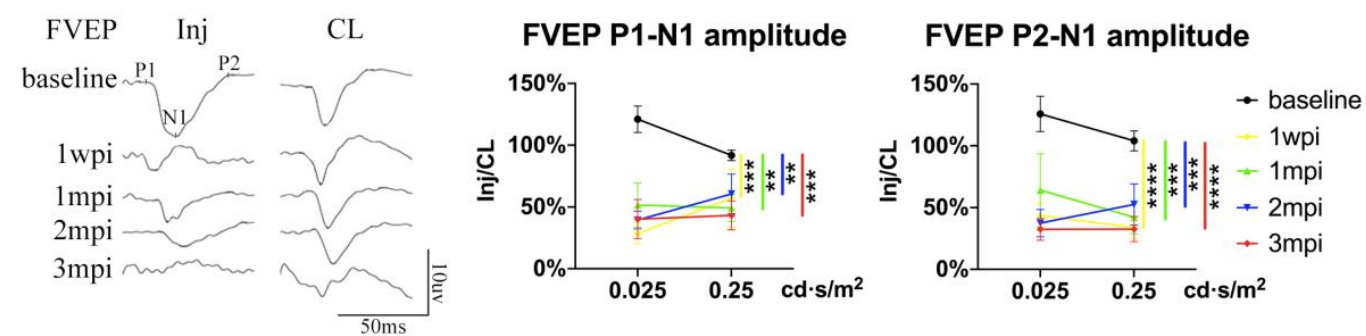

$\mathrm{E}$
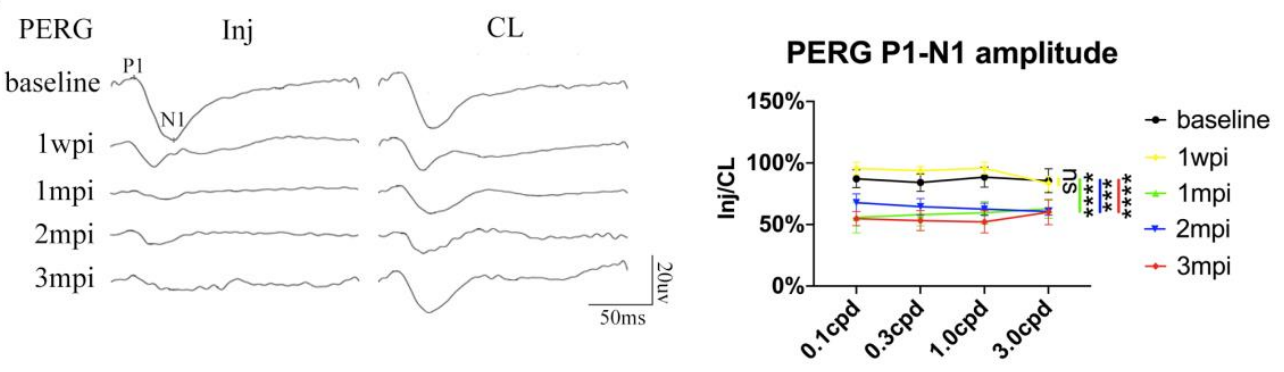

Fig. 2. Trans-nasal endoscopy-mediated pre-chiasmatic ON crush in goat leads to loss of visual function. (A) Schematic illustration of pre-chiasmatic ON exposure and crush injury. (B) Endoscopic views before (left panel) and after pre-chiasmatic ON crush (right panel). Crush site was shown in the blue circle. (C) Loss of direct pupillary light response (dPLR) in the injured eyes (left panel) and indirect PLR (iPLR) in the contralateral eyes (right panel) after crush. Friedman test with Dunn's multiple comparison (compared with 
baseline), $\mathrm{p}=0.0497$. (D) Representative FVEP waveforms of the injured eye and the contralateral eye at light intensity of $0.025 \mathrm{~cd} \cdot \mathrm{s} / \mathrm{m}^{2}$ (left panel) and quantification of FVEP P1-N1 and P2-N1 amplitudes ratios of the injured eyes to the contralateral eyes (right panel) at different time points before and after pre-chiasmatic ON crush. Two-way ANOVA with Tukey's multiple comparison (compared with the baseline). (E) Representative PERG waveforms of the injured eye and contralateral eye at spatial frequency of $0.1 \mathrm{cpd}$ (left panel) and quantification of PERG P1-N1 amplitude ratios of the injured eyes to the contralateral eyes at spatial frequencies of $0.1,0.3,1.0,3.0 \mathrm{cpd}$ (right panel) before and after pre-chiasmatic ON crush. Two-way ANOVA with Tukey's multiple comparison (compared with the baseline). Data were presented as mean \pm s.e.m, $\mathrm{n}=5$. ns: not significant, $* \mathrm{p}<0.05, * * \mathrm{p}<0.01, * * * \mathrm{p}<0.001, * * * * \mathrm{p}<0.0001$. Inj: injured, CL: contralateral, wpi: week post-injury, mpi: month post-injury. 


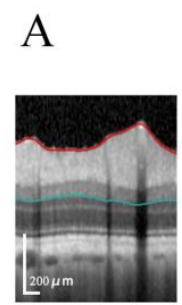

baseline

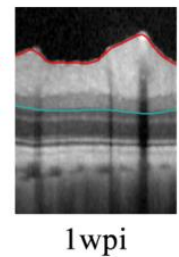

1 wpi

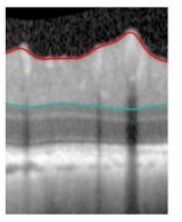

$1 \mathrm{mpi}$

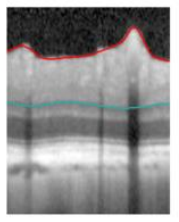

$2 \mathrm{mpi}$

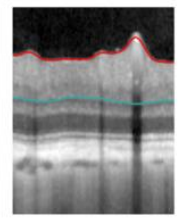

$3 \mathrm{mpi}$

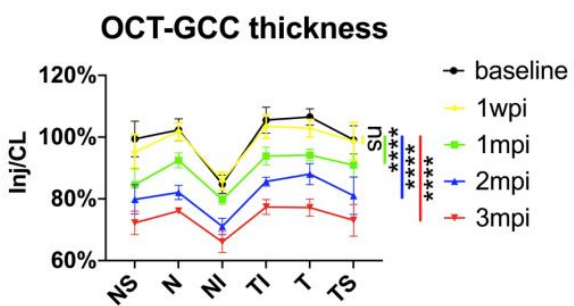

B
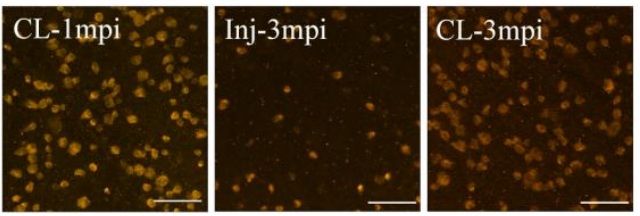

$\mathrm{C}$

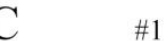

\#1 \#2 \#3

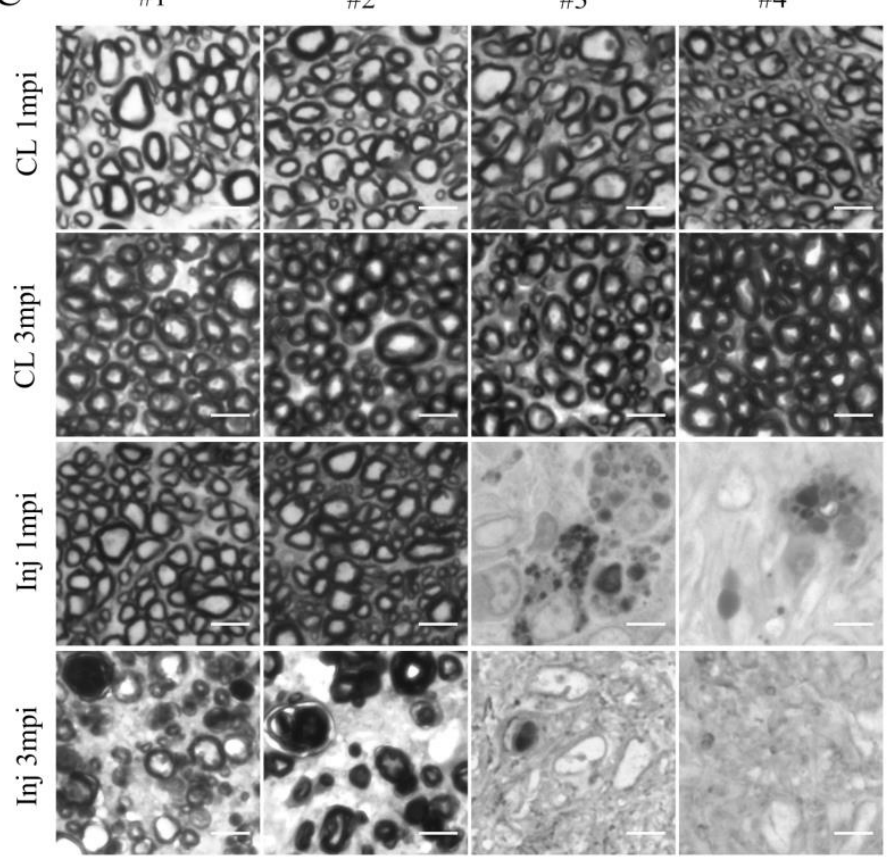

RGC density
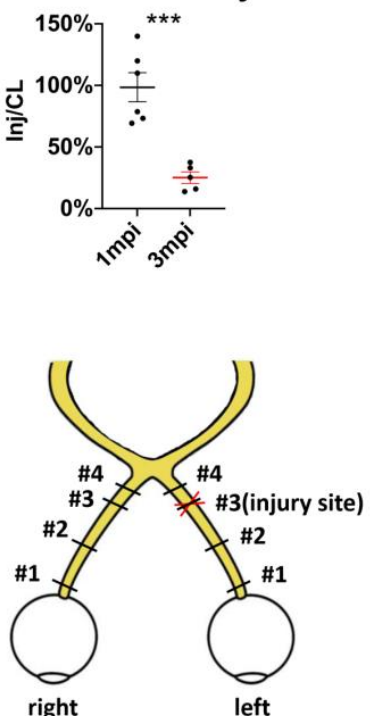

ON density

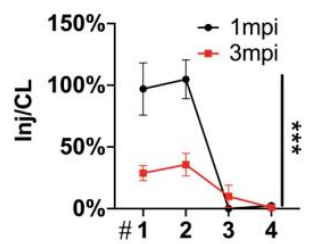


retinal flat-mounts (left panel) and quantification of RGCs densities ratios of the injured eyes to the contralateral eyes at 1, 3 mpi (right panel). n=5-6. Unpaired t-test. Scale bar $=100 \mu \mathrm{m}$. (C) Left panel: representative microscopic images of semi-thin cross sections of ON stained by PPD at different ON segments of the injured eye and its contralateral eye at 1, 3 mpi. Scale bar $=5 \mu \mathrm{m}$. Right upper panel: scheme of sampling sites at different ON segments (\#1, 2, 3, 4). Right lower panel: quantification of axonal densities ratios of the injured eyes to the contralateral eyes at different $\mathrm{ON}$ segments at 1 and 3 mpi. $n=5$. Two-way ANOVA. Data were presented as mean \pm s.e.m. ns: not significant, $* \mathrm{p}<0.05, * * \mathrm{p}<0.01$, $* * * \mathrm{p}<0.001$, **** $\mathrm{p}<0.0001$. Inj: injured, CL: contralateral, wpi: week post-injury, mpi: month post-injury. 

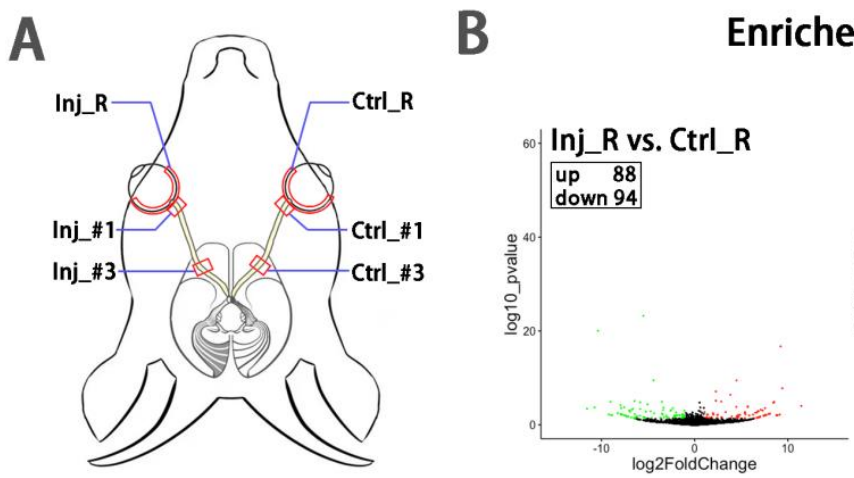

\section{Enriched Degree in the uninjured areas - No Sig • Up • Down}
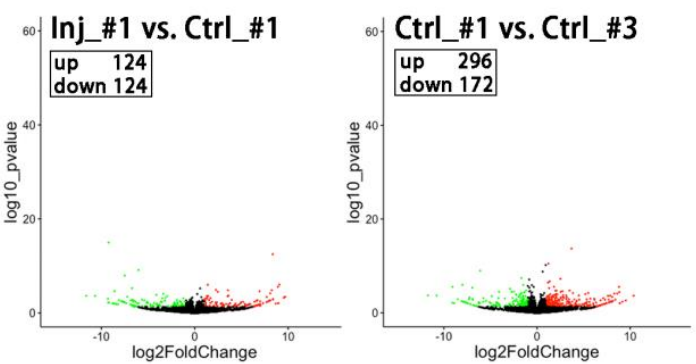

\section{Enriched Degree in the injured area}
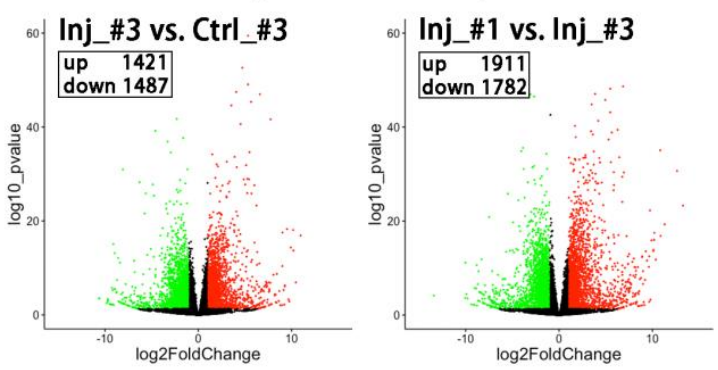

D

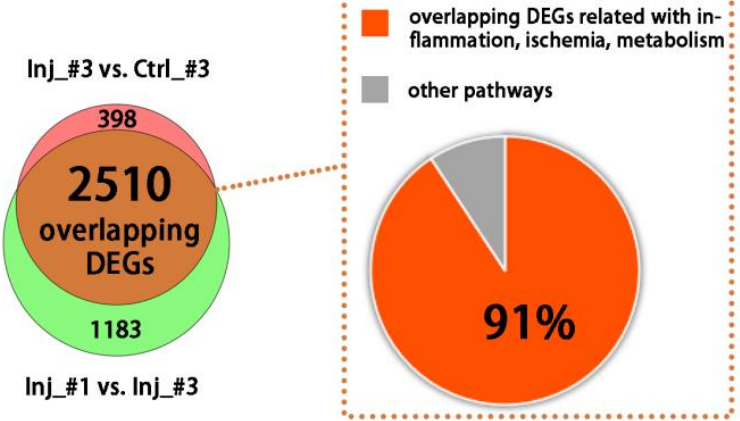

$\mathbf{E}$

Overlapping DEGs Heatmap

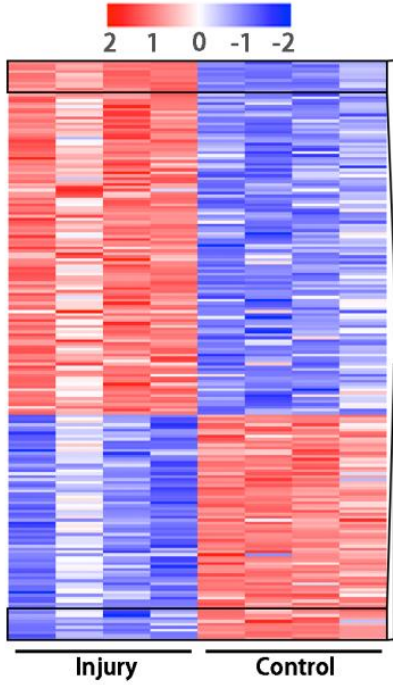

Ischemia-related

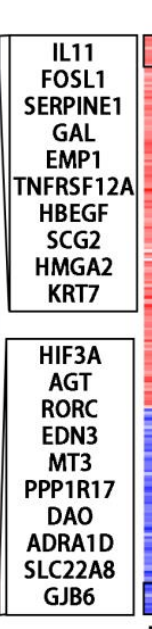

6
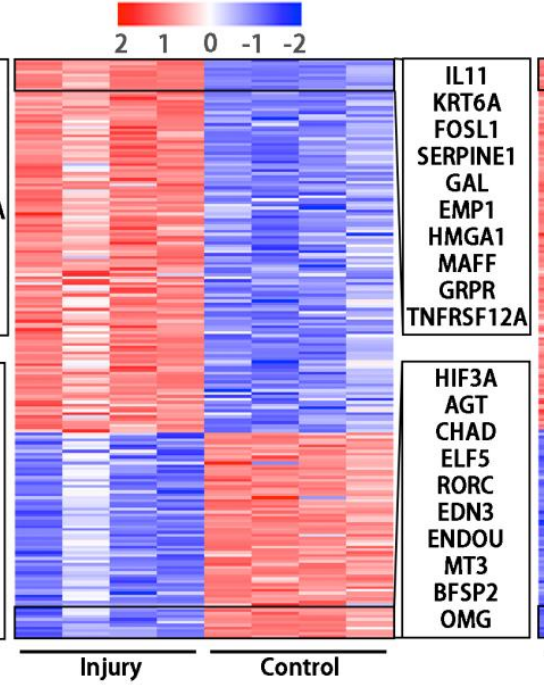

Inflammation-related

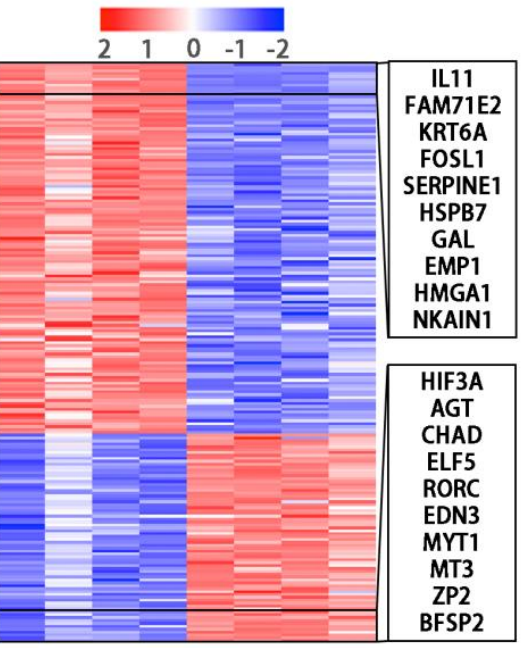

Injury Control

Metabolism-related

Fig. 4. Early transcriptomic changes are confined to the injury site and mostly enriched in the pathways of ischemia, inflammation and metabolism. (A) Scheme of sampling sites at the retina (R), retrobulbar (\#1) and pre-chiasmatic ON (\#3) of the injury eye (Inj) 
and its contralateral eye $(\mathrm{Ctrl}) .(\mathrm{B}, \mathrm{C})$ Volcano plots showing differential expression genes (DEGs) in the non-injured areas (B) and the injured areas (C). Red dots: significant upregulated genes, green dots: significant downregulated genes, adjusted $\mathrm{p}<0.05 . \log 2 \mathrm{FC}$ is 1. (D) Venn diagram indicating the overlapping DEGs between Inj_\#3 vs. Ctrl_\#3 (ipsilateral-contralateral comparison) and Inj_\#3 vs. Inj_\#1 (proximal-distal comparison of the ipsilateral eye) (left panel), $91 \%$ of which were clustered in the pathways of inflammation, ischemia and metabolism (right panel). (E): Heatmap showing TOP 200 overlapping DEGs related with pathways of ischemia (left panel), inflammation (mid panel) and metabolism (right panel) based on the DEGs of Inj_\#3 vs. Ctrl_\#3. Ranking was determined by the magnitude of fold change. Upper box shows the top 10 upregulated genes, and the below one shows the top 10 down-regulated genes. 
A

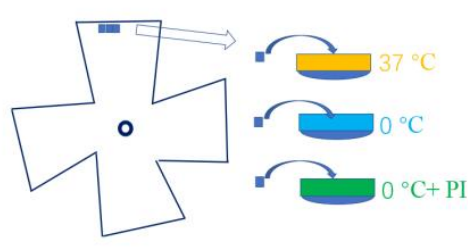

$\mathrm{D}$

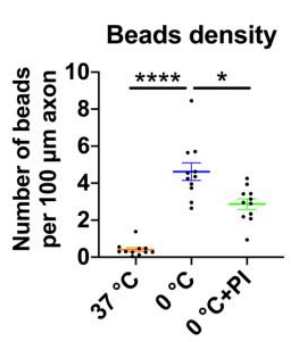

G Temperature Curve

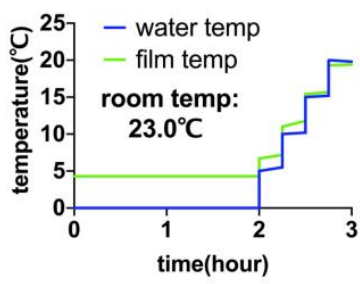

I

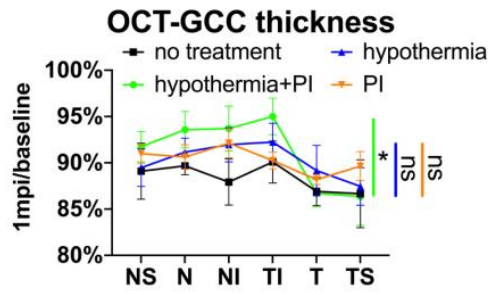

B

$\mathrm{E}$

$\mathrm{H}$

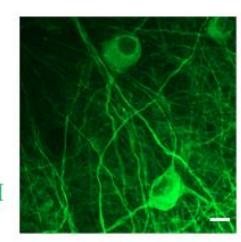

$37^{\circ} \mathrm{C}$
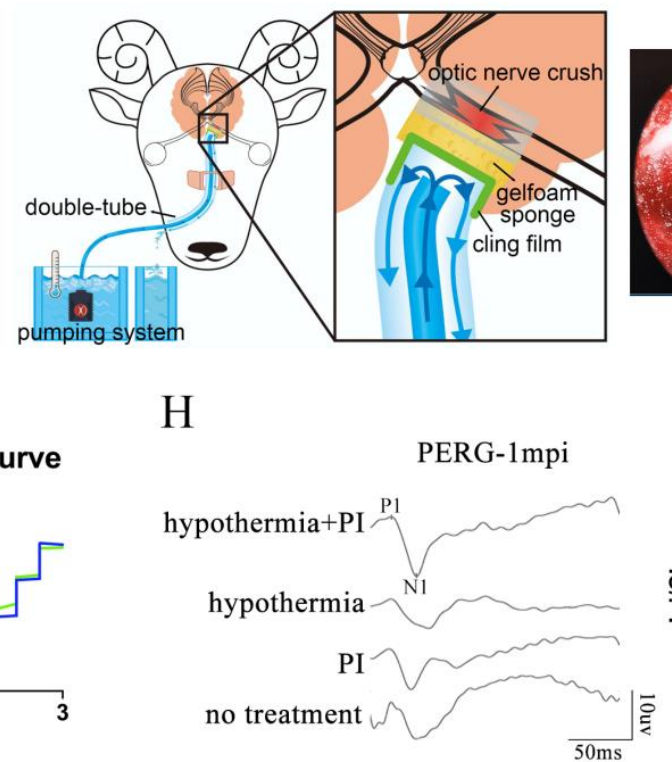

J

ON section at injury site (\#3)

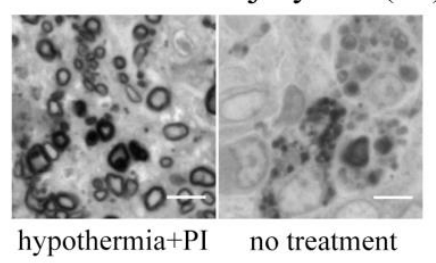

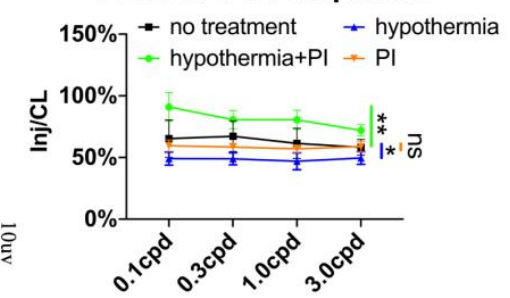

$\mathrm{C}$ cumulative axonal length

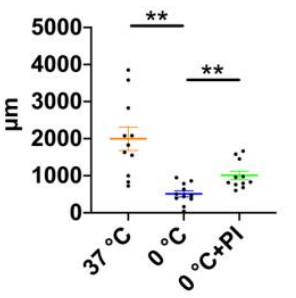
F

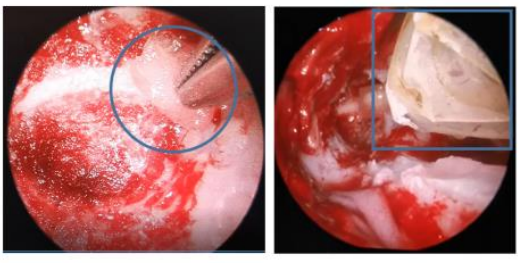

PERG P1-N1 amplitude

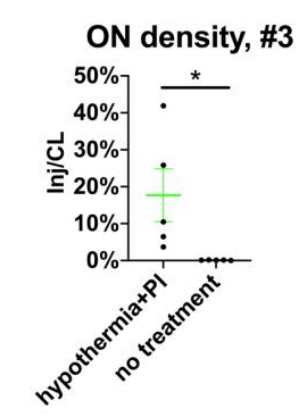
treatment alone, achieved significant neural protection. (A)Scheme for the ex vivo experiments shown in (A)- (D). (B) Representative confocal images of retinal explants immunostained with beta-3 tubulin (TUBB3) at $37^{\circ} \mathrm{C}$, at $0^{\circ} \mathrm{C}$, or at $0^{\circ} \mathrm{C}$ with PI. A typical axonal bead was labeled by a red arrow. Scale bar: $10 \mu \mathrm{m} .(C, D)$ Quantification of 
cumulative axonal length (C) and axonal beads density (D) at $37^{\circ} \mathrm{C}$, at $0^{\circ} \mathrm{C}$, and at $0^{\circ} \mathrm{C}$ with PI. n=11 retinal explants from 4 goats for each group. (E) Scheme of local delivery of hypothermia and protease inhibitors (PI) at the injury site of the pre-chiasmatic ON. (left panel) and a cooling tube (shown within a rectangle) (right panel) at the injured prechiasmatic ON. (G) Temperature curves of the cooling water and the cling film during local hypothermia. $(\mathrm{H})$ Representative PERG waveforms at spatial frequency of $0.1 \mathrm{cpd}$ (left panel) and comparison of PERG P1-N1 amplitude ratios at $1 \mathrm{mpi}$ in the following groups: hypothermia combined with PI, hypothermia alone, PI alone, no treatment. n=5-6 in each group. Two-way ANOVA, p=0.0231. (I) Comparison of GCC thickness ratio of the injured eyes to the contralateral eyes at six different regions around ON head among each group at 1 mpi. Two-way ANOVA, $\mathrm{p}=0.0478, \mathrm{n}=5-6$ in each group. (J) treatment. $\mathrm{n}=5-6$ for each group. Unpaired t-test, $\mathrm{p}=0.0398$. Data were presented as injured, CL: contralateral. 
bioRxiv preprint doi: https://doi.org/10.1101/2021.11.07.467626; this version posted November 8, 2021. The copyright holder for this preprint (which was not certified by peer review) is the author/funder, who has granted bioRxiv a license to display the preprint in perpetuity. It is made available under aCC-BY 4.0 International license.

A

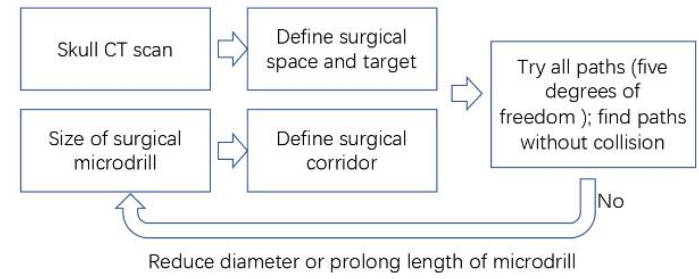

C

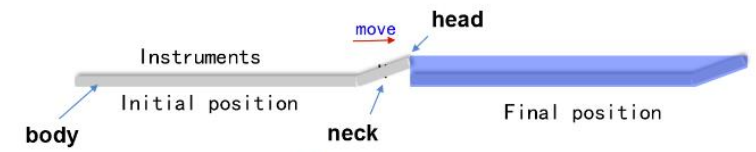

$\mathrm{D}$

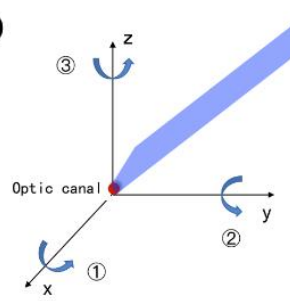

Entrance position

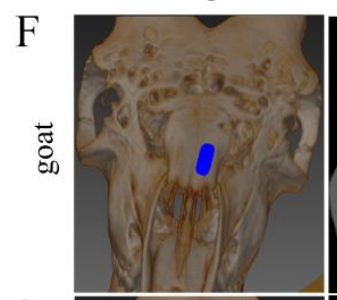

G

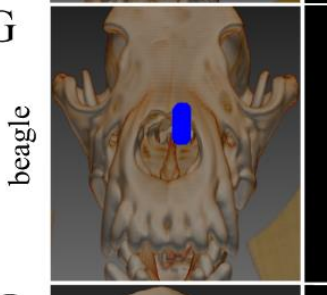

$\mathrm{H}$

$\frac{.00}{2}$

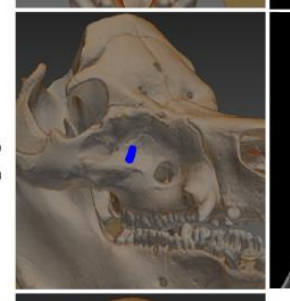

$\mathrm{I}$

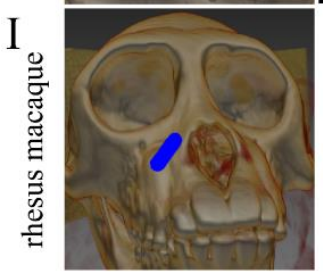

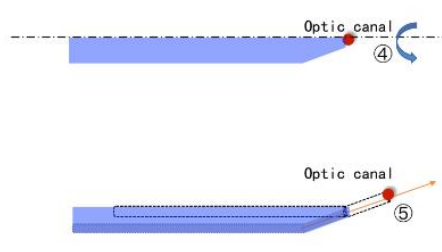

Initial position
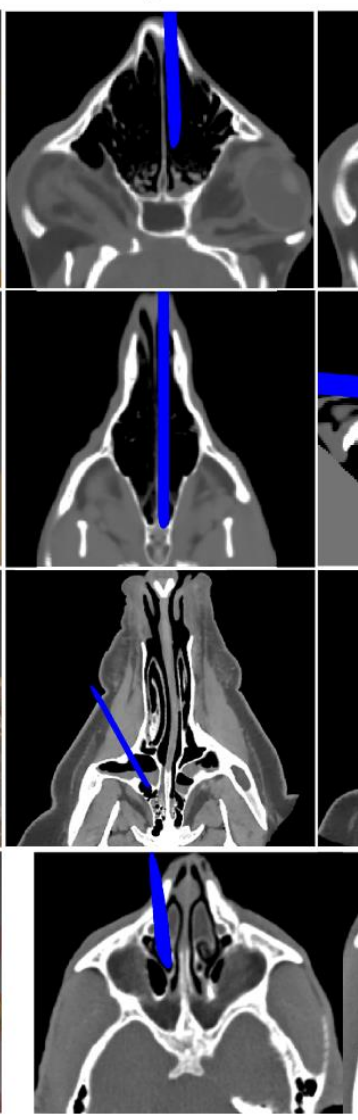

$\mathrm{B}$

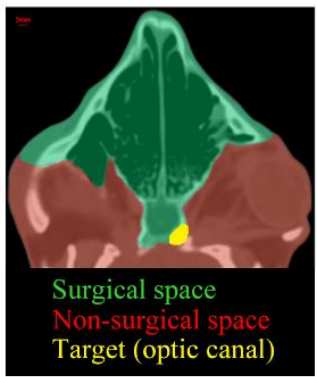

$\mathrm{E}$

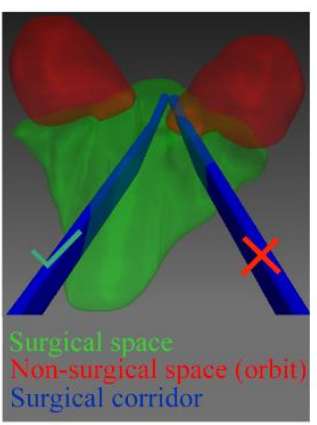

$\mathrm{J}$
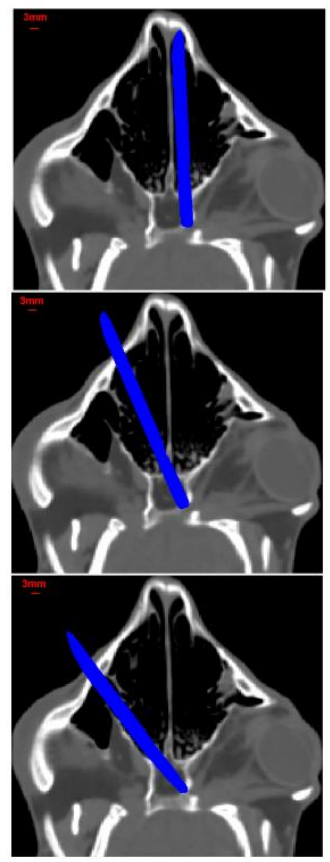

K

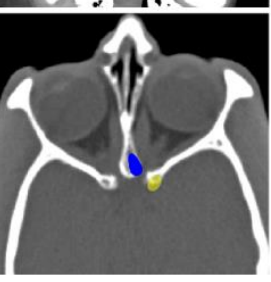

$\%$ of feasible surgical path

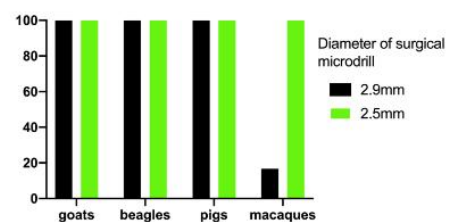




\section{Fig. 6. Computer program-aided optimization of trans-nasal endoscopic surgery in} multiple species of large animals. (A) Flowchart of the computer program. (B) Segmentation of surgical space (in green), non-surgical space (in red) and surgical target (in yellow) in the program. (C) Scheme of surgical corridor (in blue) outlined by the surgical microdrill's movement along its major axis. (D) Demonstration of five degrees of freedom of the surgical corridor. (E) Demonstration of a feasible surgical corridor (on the left) and an infeasible surgical corridor colliding with orbital cavity (on the right). (FI) Representatives of virtual surgical paths in goats, beagles, domestic pigs and rhesus macaques, respectively. Surgical entries were shown on the surface of three-dimensional reconstructed skull (left panels) and on the horizontal CT plane (middle panels). Right panels: projection of the surgical corridor on the CT plane through the pre-chiasmatic ON (surgical target). (J) Representatives of multiple feasible virtual surgical paths in goats. Scale bar $=3 \mathrm{~mm}$. (K) Quantification of percentages of feasible surgical paths in goats, beagles, domestic pigs and rhesus macaques by using different sizes of surgical microdrills $($ diameter $=2.5$ and $2.9 \mathrm{~mm})$. 


\section{Supplementary Materials}

875

876

877

878

879

880

881

882

883

884

886

887

888

Materials and Methods

Fig. S1. Endoscopic exposure of pre-chiasmatic ON is safe in goats.

Fig. S2. Contralateral eyes are functionally and structurally intact after ON crush injury.

Fig. S3. Bioinformatic analyses of the overlapping DEGs at the injury site.

Fig. S4. Bioinformatic analyses of the overlapping hub DEGs at the injury site.

Fig. S5. Local hypothermia combined with PI does not show significant rescue of PLR, FVEP and axonal density at \#4 region

Fig. S6. Pre-chiasmatic TON model in rhesus macaque

Movie S1. Surgical settings for trans-nasal endoscopic exposure of the pre-chiasmatic optic nerve in a goat.

Movie S2. Exposure of the pre-chiasmatic optic nerve via trans-nasal endoscopy in a goat.

Movie S3. Crush of the pre-chiasmatic optic nerve via trans-nasal endoscopy in a goat.

Movie S4. Trans-nasal local delivery of hypothermia and protease inhibitors to the injured prechiasmatic optic nerve in a goat.

Movie S5. Representative virtual surgical path to expose the pre-chiasmatic optic nerve in a goat.

Movie S6. Representative virtual surgical path to expose the pre-chiasmatic optic nerve in a beagle.

Movie S7. Representative virtual surgical path to expose the pre-chiasmatic optic nerve in a domestic pig. 
bioRxiv preprint doi: https://doi.org/10.1101/2021.11.07.467626; this version posted November 8, 2021. The copyright holder for this preprint

(which was not certified by peer review) is the author/funder, who has granted bioRxiv a license to display the preprint in perpetuity. It is made available under aCC-BY 4.0 International license.

894 Movie S8. Representative virtual surgical path to expose the pre-chiasmatic optic nerve in a

895 rhesus macaque.

896 Movie S9. Exposure of the pre-chiasmatic optic nerve via trans-nasal endoscopy in a rhesus

897 macaque. 


\section{Supplementary Materials:}

A

OCT-GCC thickness, CL

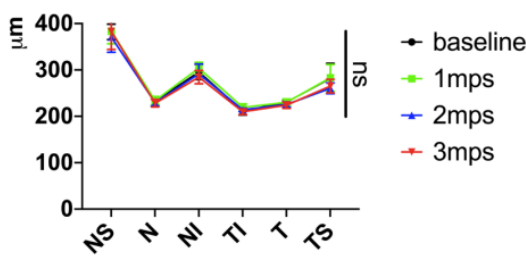

$\mathrm{C}$

D

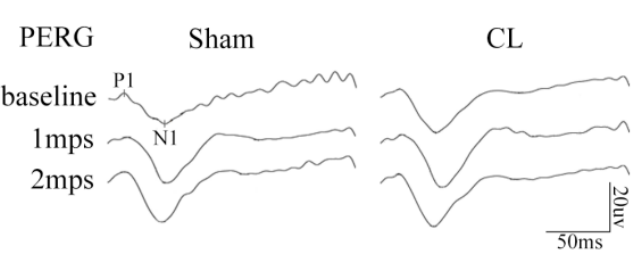

B

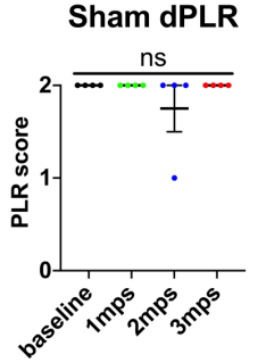

CL iPLR

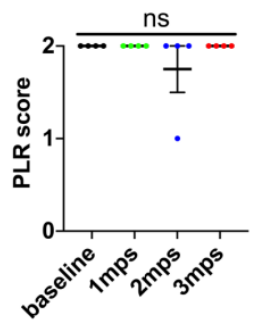

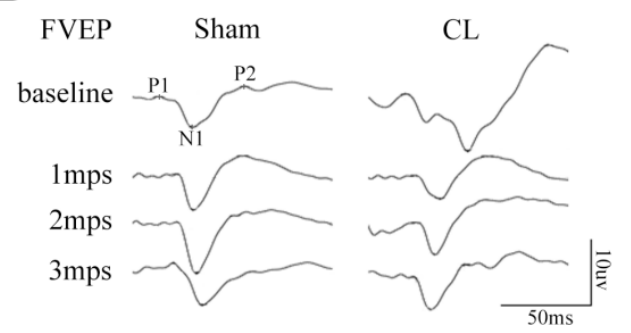

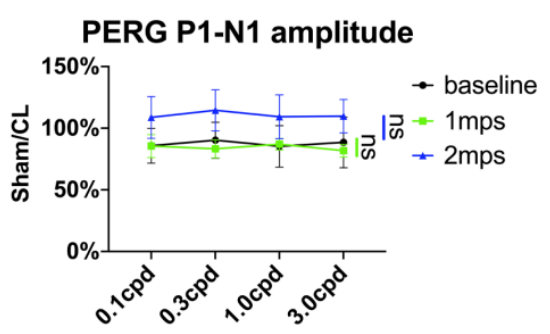

Figure S1. Endoscopic exposure of pre-chiasmatic ON is safe in goats.

(A) Quantification of GCC thickness of the contralateral eyes before and after pre-chiasmatic

ON exposure in goats. Two-way ANOVA with Tukey's multiple comparison (compared with baseline). $n=4$. (B) Quantification of direct pupillary light response (dPLR) in the sham surgical eyes (left panel) and indirect PLR (iPLR) in the contralateral eyes (right panel) after prechiasmatic ON exposure. n=4. Friedman test with Dunn's multiple comparison (compared with baseline), $p>0.9999$. (C) Representative PERG waveforms of the sham surgery eye and the contralateral eye at spatial frequency of $0.1 \mathrm{cpd}$ (left panel) and quantification of P1-N1 amplitude ratios of PERG in the sham surgical eyes to the contralateral eyes (right panel) before 
and after pre-chiasmatic ON exposure. n=4. Two-way ANOVA with Sidak's multiple comparison (compared with the baseline). (D) Representative FVEP waveforms of the sham surgical eyes and the contralateral eyes at light intensity of $0.025 \mathrm{~cd} \cdot \mathrm{s} / \mathrm{m}^{2}$ (left panel) and quantification of P1-N1 and P2-N1 amplitudes ratios of FVEP in the sham surgical eyes to the contralateral eyes at light intensities of 0.025 and $0.25 \mathrm{~cd} \cdot \mathrm{s} / \mathrm{m}^{2}$ (right panel) before and after prechiasmatic ON exposure. n=3-4. Two-way ANOVA with Sidak's multiple comparison (compared with the baseline). Data were presented as mean \pm s.e.m. ns: not significant, CL: contralateral, mps: month post-surgery (post sham surgery).

A goats, ON crush

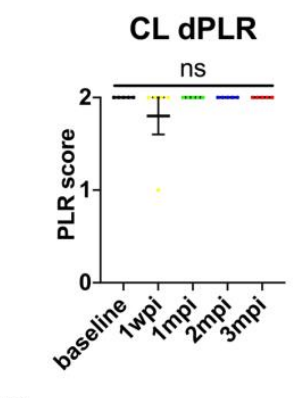

B goats, ON crush

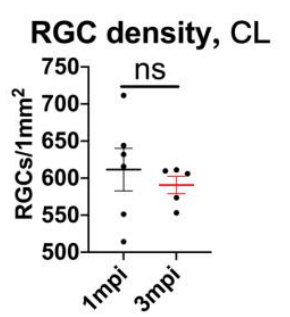

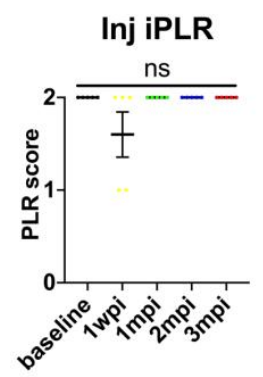

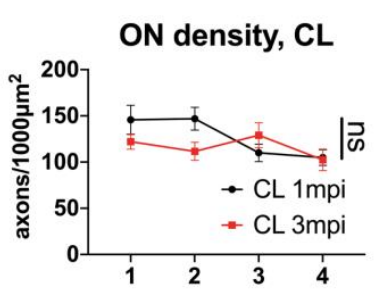

OCT-GCC thickness, CL

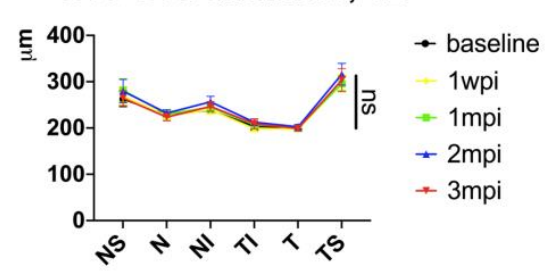

$\mathrm{C}$ rhesus macaques, ON crush

OCT-GCC thickness, CL

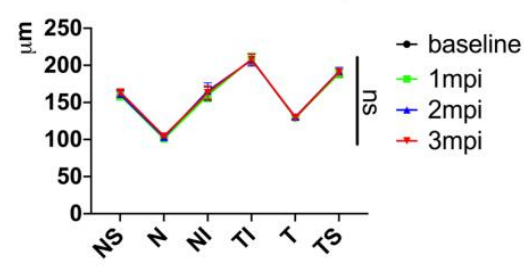

Figure S2. Contralateral eyes are functionally and structurally intact after ON crush

injury. (A) Left panel: Quantification of dPLR in the contralateral eyes and iPLR in the injured eyes after pre-chiasmatic ON crush in goats. Friedman test with Dunn's multiple comparison (compared with baseline). Right panel: quantification of GCC thickness of the contralateral eyes 
after pre-chiasmatic ON crush in goats. Two-way ANOVA with Tukey's multiple comparison (compared with baseline). n=5. (B) Left panel: quantification of RGCs densities of the contralateral eyes at 1, 3 mpi after pre-chiasmatic ON crush in goats. Unpaired t-test. $\mathrm{n}=5-6$.

Right panel: quantification of axonal densities of the contralateral eyes at different ON segments at 1, 3 mpi after pre-chiasmatic ON crush in goats. Two-way ANOVA. n=3-6. (C) Quantification of GCC thickness of the contralateral eyes after pre-chiasmatic ON crush in rhesus macaques.

Two-way ANOVA with Tukey's multiple comparison (compared with baseline). $n=4$. Data were presented as mean \pm s.e.m, ns: not significant, CL: contralateral, wpi: week post-injury, mpi: month post-injury.
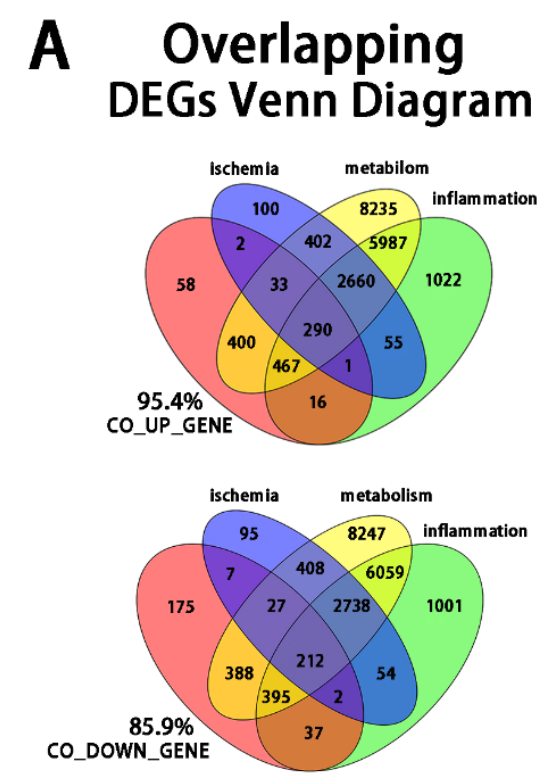

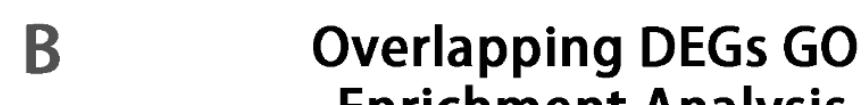
Enrichment Analysis

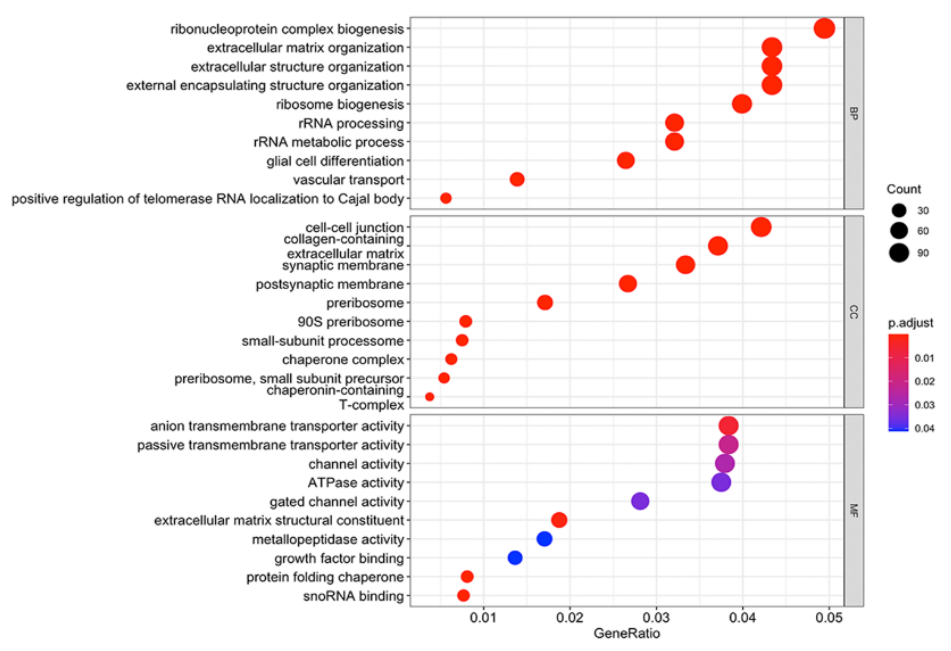

Figure S3. Bioinformatic analyses of the overlapping DEGs at the injury site

(A) Venn diagram showing that $95.4 \%$ of the up-regulated and $85.9 \%$ of the down-regulated overlapping DEGs at the injury sit were enriched in the pathways of ischemia, inflammation, metabolism. 
(B) Enrichment analysis of overlapping DEGs using the Gene Ontology.

\section{A TOP50 Overlapping Hub DEGs}

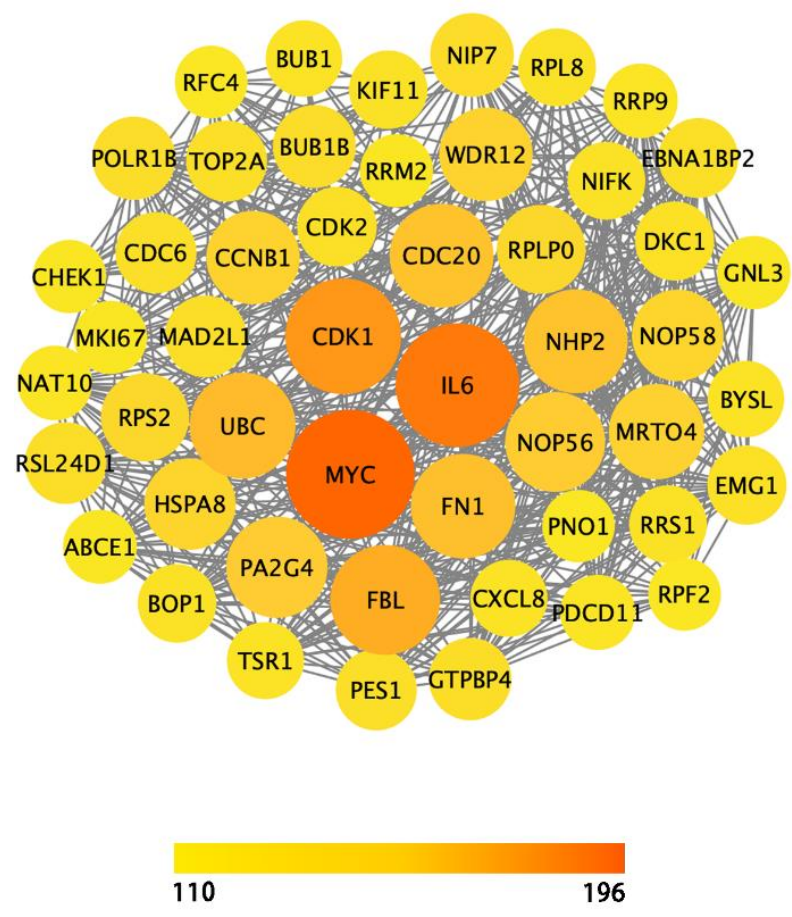

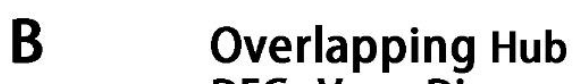
DEGs Venn Diagram

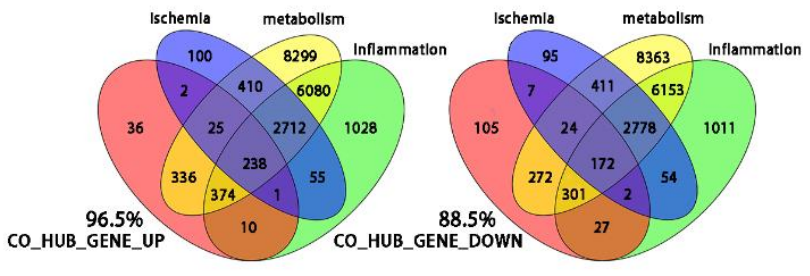

\section{Overlapping Hub DEGs GO Enrichment Analysis}

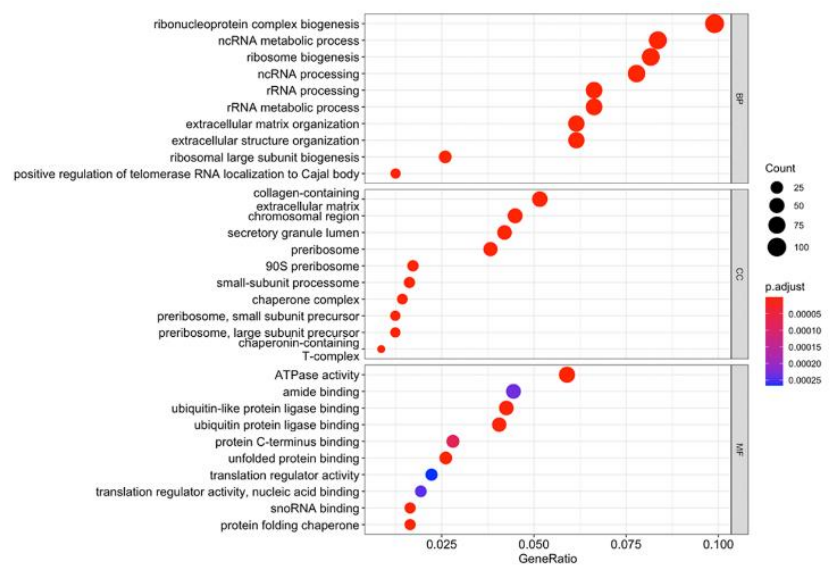

D

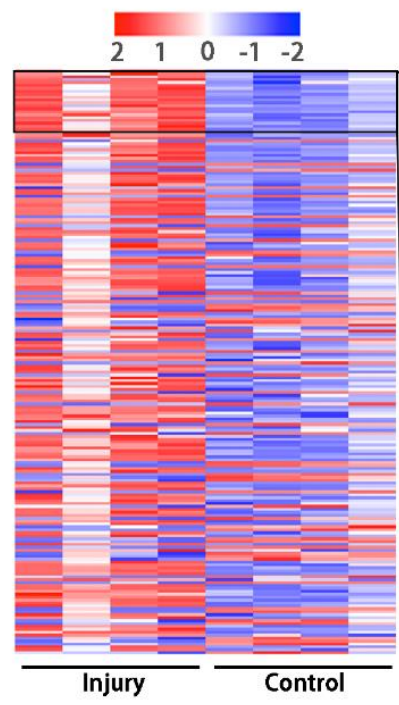

Ischemia-related

\section{Overlapping Hub DEGs Heatmap}
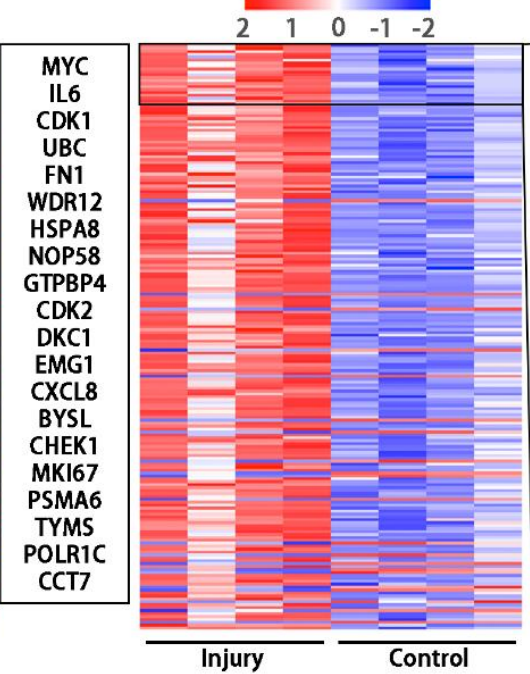

\begin{tabular}{||c|}
\hline MYC \\
IL6 \\
CDK1 \\
FBL \\
UBC \\
FN1 \\
NHP2 \\
PA2G4 \\
CCNB1 \\
HSPA8 \\
NOP58 \\
RPLP0 \\
RPS2 \\
CDC6 \\
TOP2A \\
CDK2 \\
DKC1 \\
KIF11 \\
CXCL8 \\
BYSL \\
\hline
\end{tabular}

Inflammation-related

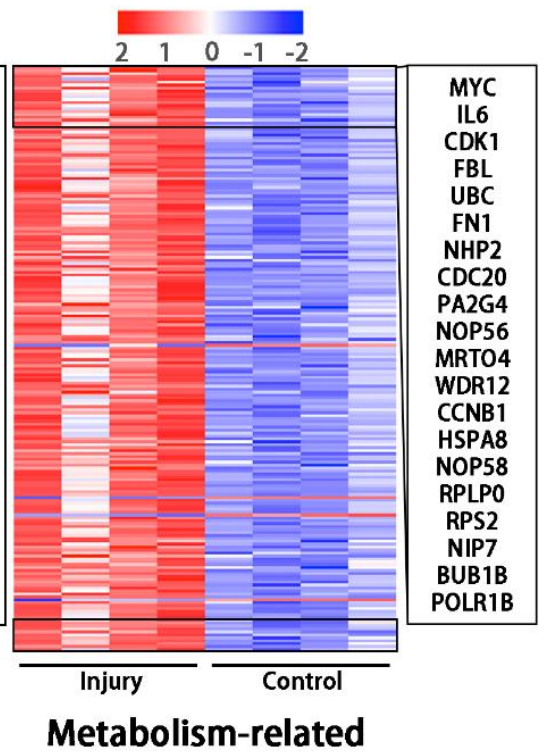




\section{Figure S4. Bioinformatic analyses of the overlapping hub DEGs at the injury site}

(A) Protein-protein interactions diagram of top 50 overlapping hub gene (DEGs with highest connectivity). (B) Venn diagram showing that $96.5 \%$ of the up-regulated and $88.5 \%$ of the down-regulated hub genes were enriched in the pathways of ischemia, inflammation, metabolism. (C) Enrichment analysis of hub genes using the Gene Ontology (GO). (D) Heatmap showing TOP overlapping hub genes enriched in the pathways of ischemia (left panel), inflammation (mid panel) and metabolism (right panel) based on the hub genes of Inj_\#3 vs. Ctrl_\#3 group. Ranking was determined by the magnitude of fold change.

A Inj dPLR

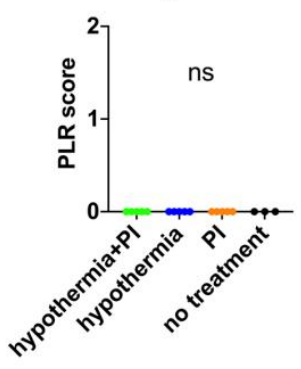

$\mathrm{C}$

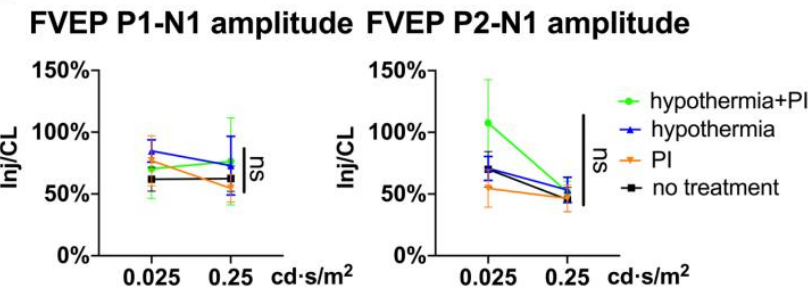

B

\section{IPLR}

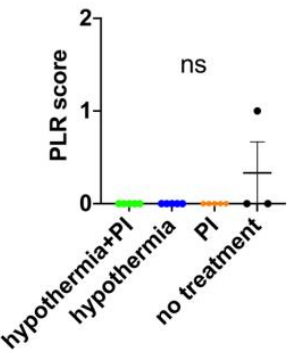

FVEP $\quad 0.025 \mathrm{~cd} \cdot \mathrm{s} / \mathrm{m}^{2} \quad 0.25 \mathrm{~cd} \cdot \mathrm{s} / \mathrm{m}^{2}$

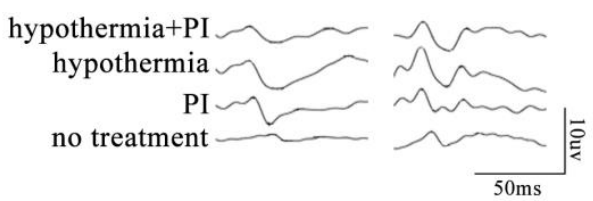

D

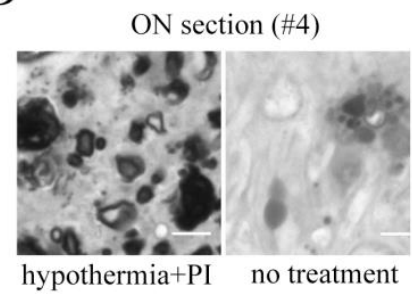

ON density, \#4

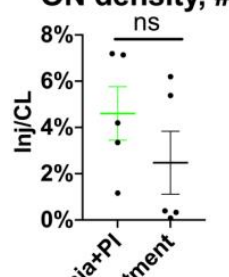

Figure S5. Local hypothermia combined with PI does not show significant rescue of PLR, FVEP, and axonal density at distal ON segment (\#4 region) 
(A) Quantification of dPLR in the injured eyes (left panel) and iPLR in the contralateral eyes (right panel) in the groups of hypothermia combined with PI, hypothermia alone, PI alone and no treatment. $n=5-6$ in each group. $(B, C)$ Representative FVEP waveform and quantification of the $\mathrm{P} 1-\mathrm{N} 1$ and P2-N1 amplitudes ratios of FVEP in the injured eyes to the contralateral eyes in each group at 1 mpi. $n=5-6$ in each group. Two-way ANOVA. (D) Representative images of ON semi-section (left panel) and comparison of axonal densities (right panel) in the groups of hypothermia combined with PI and no treatment at the \#4 segment at $1 \mathrm{mpi} . \mathrm{n}=5-6$ for each group. Unpaired t-test. Data were presented as mean \pm s.e.m. ns: not significant. Inj: injured, CL: contralateral. 
bioRxiv preprint doi: https://doi.org/10.1101/2021.11.07.467626; this version posted November 8, 2021. The copyright holder for this preprint (which was not certified by peer review) is the author/funder, who has granted bioRxiv a license to display the preprint in perpetuity. It is made available under aCC-BY 4.0 International license.

A

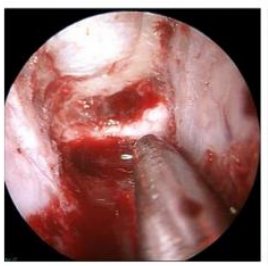

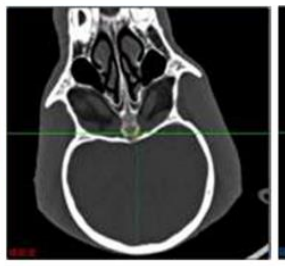

CL

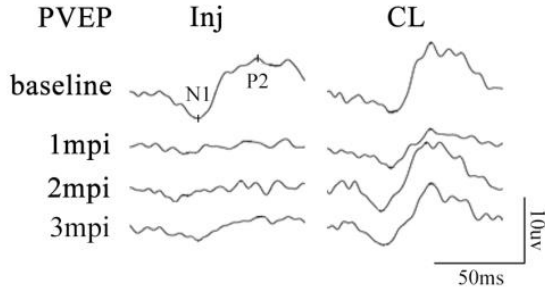

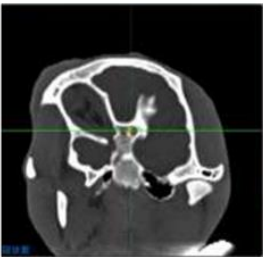

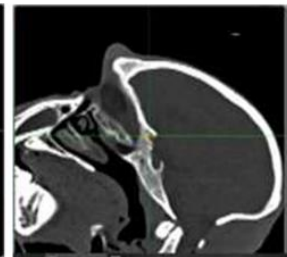

$\mathrm{B}$

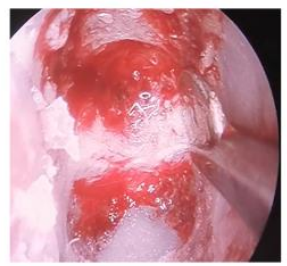

C

$\mathrm{D}$

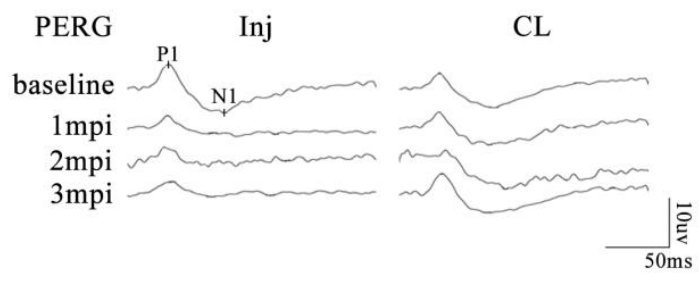

PVEP N1-P2 amplitude

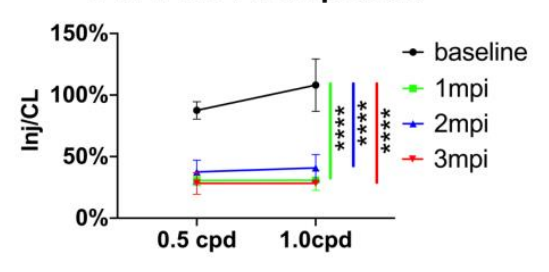

$\mathrm{E}$

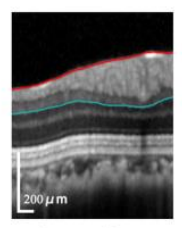

baseline

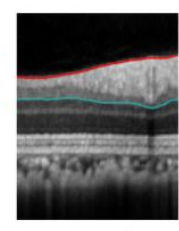

1 wpi

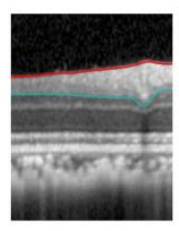

$1 \mathrm{mpi}$

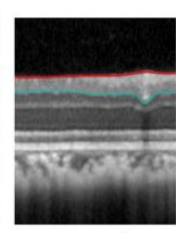

$2 \mathrm{mpi}$
PERG P1-N1 amplitude

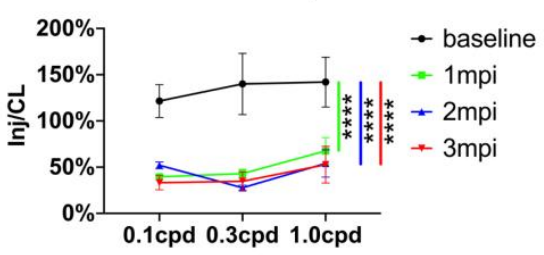

$\mathrm{F}$
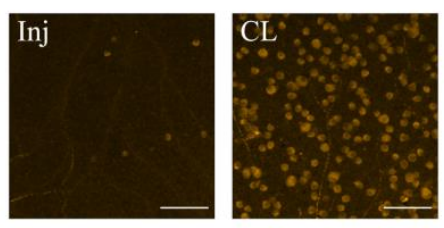

RGC density
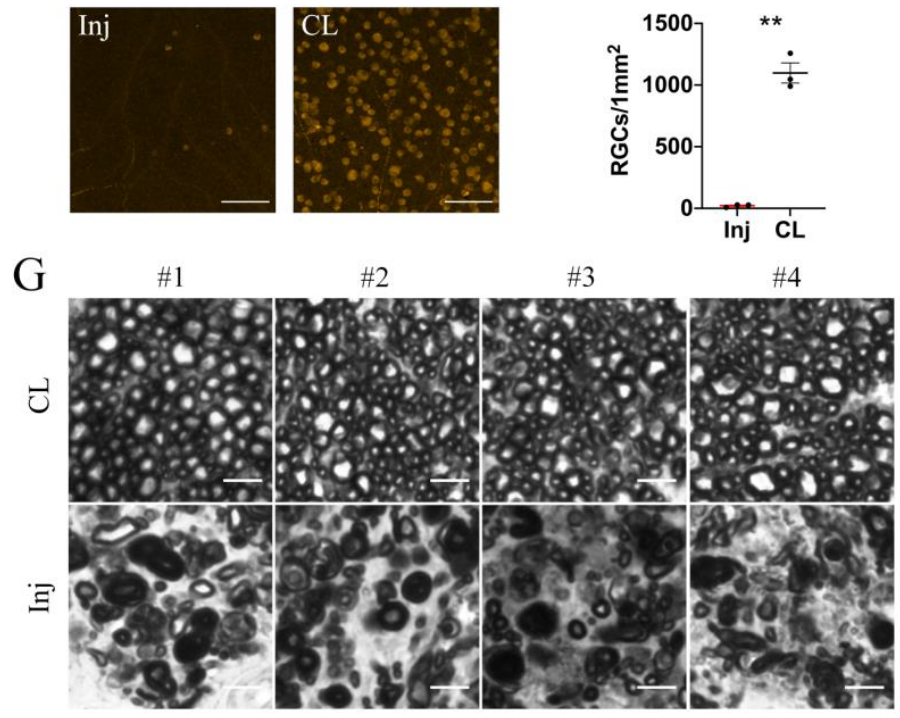

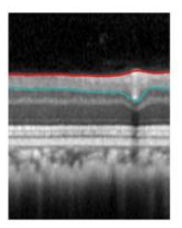

$3 \mathrm{mpi}$

\section{OCT-GCC thickness}

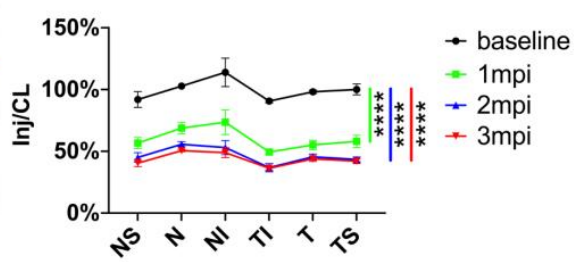




\section{Figure S6. Pre-chiasmatic TON model in rhesus macaque}

(A) Confirmation of the pre-chiasmatic $\mathrm{ON}$ in rhesus macaques (left panel) using the surgical navigation system on the horizontal, coronal and sagittal CT planes (right panel). (B) Endoscopic image of pre-chiasmatic ON crush. (C) Representative PVEP waveforms of the injured eye and the contralateral eye at spatial frequencies of $0.5 \mathrm{cpd}$ (left panel) and quantification of the N1-P2 amplitude ratios of the injured eyes to the contralateral eyes at spatial frequencies of 0.5 and 1.0 cpd before and after crush (right panel). n=4. Two-way ANOVA with Tukey's multiple comparison (compared with the baseline). (D) Representative PERG waveforms of the injured eye and the contralateral eye at spatial frequency of $0.1 \mathrm{cpd}$ (left panel) and quantification of P1$\mathrm{N} 1$ amplitude ratios of the injured eyes to the contralateral eyes at spatial frequencies of $0.1,0.3$, $1.0 \mathrm{cpd}$ (right panel) before and after crush. n=3-4. Two-way ANOVA with Tukey’s multiple comparison (compared with the baseline). (E) Representative OCT images of the injured eye (left panel) and quantification of GCC thickness ratios of the injured eyes to the contralateral eyes at six regions around $\mathrm{ON}$ head before and after crush (right panel). $\mathrm{n}=4$. Two-way ANOVA with Tukey's multiple comparison (compared with the baseline). (F) Representative immunostaining images of the RBMPS positive RGCs in the retinal flat-mounts (left panel) and quantification of RGCs densities of the injured eyes and the contralateral eyes at 3 mpi (right panel). $n=3$. Paired t-test. Scale bar $=100 \mu \mathrm{m}$. (G) Left panel: representative microscopic images of semi-thin cross sections of ON stained by PPD at different ON segments $(\# 1,2,3,4)$ of the injured eyes and its contralateral eyes at 3 mpi. Scale bar $=5 \mu \mathrm{m}$. Right panel: comparison of axonal densities at different $\mathrm{ON}$ sites between the injured eyes and the contralateral eyes at 3 mpi. $n=3$. Two-way ANOVA. Data were presented as mean \pm s.e.m. ns: not significant, *:p<0.05, $* *: \mathrm{p}<0.01, * * *: \mathrm{p}<0.001, * * * *: \mathrm{p}<0.0001$. Inj: injured, CL: contralateral, mpi: month post-injury. 
Movie S1. Surgical settings for trans-nasal endoscopic exposure of the pre-chiasmatic optic nerve in a goat.

Movie S2. Exposure of the pre-chiasmatic optic nerve via trans-nasal endoscopy in a goat.

Movie S3. Crush of the pre-chiasmatic optic nerve via trans-nasal endoscopy in a goat.

Movie S4. Trans-nasal local delivery of hypothermia and protease inhibitors to the injured prechiasmatic optic nerve in a goat.

Movie S5. Representative virtual surgical path to expose the pre-chiasmatic optic nerve in a goat.

Movie S6. Representative virtual surgical path to expose the pre-chiasmatic optic nerve in a beagle.

Movie S7. Representative virtual surgical path to expose the pre-chiasmatic optic nerve in a domestic pig.

Movie S8. Representative virtual surgical path to expose the pre-chiasmatic optic nerve in a rhesus macaque.

Movie S9. Exposure of the pre-chiasmatic optic nerve via trans-nasal endoscopy in a rhesus macaque. 